\title{
Harmonic volume and its applications
}

\author{
Yuuki Tadokoro \\ Natural Science Education, \\ Kisarazu National College of Technology, 2-11-1 Kiyomidai-Higashi, \\ Kisarazu, Chiba 292-0041, Japan \\ email:tado@nebula.n.kisarazu.ac.jp
}

\begin{abstract}
The period is a classical complex analytic invariant for a compact Riemann surface defined by integration of differential 1-forms. It has a strong relationship with the complex structure of the surface. In this chapter, we review another complex analytic invariant called the harmonic volume. It is a natural extension of the period defined using Chen's iterated integrals and captures more detailed information of the complex structure. It is also one of a few explicitly computable examples of complex analytic invariants. As an application, we give an algorithm in proving nontriviality for a class of homologically trivial algebraic cycles obtained from special compact Riemann surfaces. The moduli space of compact Riemann surfaces is the space of all biholomorphism classes of compact Riemann surfaces. The harmonic volume can be regarded as an analytic section of a local system on the moduli space. It enables a quantitative study of the local structure of the moduli space. We explain basic concepts related to the harmonic volume and its applications of the moduli space.
\end{abstract}

2000 Mathematics Subject Classification: 14H40, 14H50, 30F30, 32 G15.

Keywords: Riemann surface, Harmonic volume, Iterated integral.

\section{Contents}

1 Introduction . . . . . . . . . . . . . . . . 2

2 Preliminaries ......................... 6

2.1 Iterated integrals . . . . . . . . . . . . . . 6

2.2 Harmonic volume . . . . . . . . . . . . . . . . 9

2.3 The Chow group . . . . . . . . . . . . . . 13

3 Abel-Jacobi maps and harmonic volume . . . . . . . . . . . . . . 15

4 Pointed Torelli Theorem . . . . . . . . . . . . . . . . 18

5 Nontrivial algebraic cycles in the Jacobian varieties . . . . . . . . 21 
$5.1 \quad$ Fermat curves . . . . . . . . . . . . . . . . 21

5.2 A generalized hypergeometric function . . . . . . . . . . 24

5.3 Cyclic quotients of Fermat curves . . . . . . . . . . . 26

\section{Introduction}

Let $\Gamma_{g}$ be the mapping class group of an oriented closed surface $\Sigma_{g}$ of genus $g \geq 2$. This is the group of all isotopy classes of orientation-preserving diffeomorphisms of $\Sigma_{g}$. The moduli space $\mathcal{M}_{g}=\mathcal{T}_{g} / \Gamma_{g}$ of compact Riemann surfaces of genus $g$ is the quotient space of the Teichmüller space $\mathcal{T}_{g}$ by the natural action of the mapping class group $\Gamma_{g}$. It is the space of all biholomorphism classes of compact Riemann surfaces of genus $g$. The global structure of $\mathcal{M}_{g}$ is one of the most attractive subjects in mathematics. For its study, topological methods such as cohomology and homotopy theory are often used. This comes from the well-known fact that the natural properly discontinuous action of the mapping class group on the Teichmüller space $\mathcal{T}_{g}$ which is contractible yields a rational cohomology equivalence between the moduli space $\mathcal{M}_{g}$ and the classifying space of the mapping class group $\Gamma_{g}$.

In contrast, for the analytic approach, the universal family $\pi: \mathcal{C}_{g} \rightarrow \mathcal{M}_{g}$ of compact Riemann surfaces of genus $g$ plays an important role. The Abel-Jacobi map from a compact Riemann surface to its Jacobian variety has been studied by many people. Here we focus on the fact that the Abel-Jacobi map can be regarded as a natural section of a local system on $\mathcal{C}_{g}$. The pointed harmonic volume, the main theme of this chapter, is an extension of this framework. We review the pointed harmonic volume and its applications.

Let $H$ be the set of real harmonic 1-forms on a compact Riemann surface with integral periods. The Hodge theorem gives an identification of $H$ with the first integral cohomology group of $\Sigma_{g}$. Let $k \geq 0$ be an integer. The natural action of $\Gamma_{g}$ on $H$ gives a $\Gamma_{g}$-module structure on $\operatorname{Hom}_{\mathbb{Z}}\left(\wedge^{2 k+1} H, \mathbb{R} / \mathbb{Z}\right)$, where $\wedge^{2 k+1}$ is the $(2 k+1)$-th wedge product and the action on $\mathbb{R} / \mathbb{Z}$ is trivial. This $\Gamma_{g}$-module gives rise to a local system $\mathcal{L}_{k}$ of free abelian groups on $\mathcal{M}_{g}$ by a pullback along $\pi$ on the universal family $\mathcal{C}_{g}$. We consider two natural sections of $\mathcal{L}_{k}$. The first is the well-known Abel-Jacobi map $A: \mathcal{C}_{g} \rightarrow \mathcal{L}_{0}$. Let $X$ be a compact Riemann surface of genus $g \geq 2$ and $x \in X$ a base point.

The Abel-Jacobi map $A_{x}: X \rightarrow \operatorname{Hom}(H, \mathbb{R} / \mathbb{Z})$ is defined by

$$
A_{x}: X \ni y \mapsto\left(\omega \mapsto \int_{x}^{y} \omega\right) \in \operatorname{Hom}(H, \mathbb{R} / \mathbb{Z}) .
$$

The second is the pointed harmonic volume

$$
I: \mathcal{C}_{g} \rightarrow \mathcal{L}_{1}
$$


studied by Harris [17] and Pulte [28]. These two natural sections uniformly can be interpreted as an integration on the $(2 k+1)$-th chain in the Jacobian variety of $X$. See Section 3 for more details. We focus on the latter. We remark that a key object is the module $\operatorname{Hom}_{\mathbb{Z}}\left(\wedge^{3} H, \mathbb{R} / \mathbb{Z}\right)$.

We define the pointed harmonic volume for a pointed compact Riemann surface $(X, x)$. Let $K$ denote the kernel of $():, H \otimes H \rightarrow \mathbb{Z}$ induced by the intersection pairing. For $\sum_{i=1}^{n} a_{i} \otimes b_{i} \in K$ and $c \in H$, the pointed harmonic volume is defined to be a homomorphism $I_{(X, x)}: K \otimes H \rightarrow \mathbb{R} / \mathbb{Z}$ given by

$$
I_{(X, x)}\left(\left(\sum_{i=1}^{n} a_{i} \otimes b_{i}\right) \otimes c\right)=\sum_{i=1}^{n} \int_{\gamma} a_{i} b_{i}+\int_{\gamma} \eta \bmod \mathbb{Z} .
$$

Here the integral $\int_{\gamma} a_{i} b_{i}$ is Chen's iterated integral, $\gamma$ is a loop in $X$ with base point $x$ whose homology class is the Poincaré dual of $c$, and $\eta$ is a unique smooth 1-form on $X$ satisfying the two conditions $d \eta+\sum_{i=1}^{n} a_{i} \wedge b_{i}=0$ and $\int_{X} \eta \wedge * \alpha=0$ for any closed 1-form $\alpha$ on $X$. Through a natural homomorphism $K \otimes H \rightarrow \wedge^{3} H$, the pointed harmonic volume $I_{(X, x)}$ can be regarded as an element of $\operatorname{Hom}_{\mathbb{Z}}\left(\wedge^{3} H, \mathbb{R} / \mathbb{Z}\right)$.

The pointed harmonic volume has been studied in the context of, for example, algebraic geometry, number theory, complex analysis, and topology. It is defined using Chen's [6] iterated integrals, which were originally used in the context of differential geometry on loop spaces. The pointed harmonic volume has the following three features. First, as an extension of the Abel-Jacobi map, the pointed harmonic volume captures the geometric information of pointed compact Riemann surfaces. Actually, it induces the mixed Hodge structure of the truncation on the fundamental group ring of a pointed compact Riemann surface. Using this, we obtain the so-called pointed Torelli theorem stating that the pointed harmonic volume determines the structure of the moduli space of pointed compact Riemann surfaces. Second, the pointed harmonic volume is explicitly computable for a number of special compact Riemann surfaces including the Fermat curves. In fact, there are not so many computable analytic invariants. The pointed harmonic volume enables a quantitative study of the local structure of $\mathcal{M}_{g}$ and $\mathcal{C}_{g}$. For example, we explain the nontriviality of the Ceresa cycle, see Section 2.3, in the Jacobian varieties of compact Riemann surfaces. Third, we can also compute the first variation of the pointed harmonic volume. It is a twisted 1 -form on $\mathcal{C}_{g}$ representing the first extended Johnson homomorphism [24] on the mapping class group of a pointed oriented closed surface. In other words, the first variation represents the first Morita-Mumford class or tautological class $e_{1} \in H^{2}\left(\mathcal{M}_{g} ; \mathbb{Z}\right)$ [23, 25]. From the viewpoint of Hodge theory, Hain and Reed [15] obtained a similar result. Since Kawazumi [20] has reviewed the last of these, we make no mention of it here. See Kawazumi's chapter [20] for more details. We remark that few values of the first variation of the harmonic volume for a specific compact Rie- 
mann surface are known. For yet another method for the harmonic volume, we recommend the survey 14 by Hain.

In the following, we overview the theory of the harmonic volume. More details will be given in subsequent sections. Harris [17] defined the harmonic volume for compact Riemann surfaces using Chen's [6] iterated integrals of 1-forms of length 2. See Section 2.1 for the iterated integrals and 2.2 for the harmonic volume. Prior to the harmonic volume, Harris [16] studied an explicit formula of triple products of holomorphic cusp forms for $\operatorname{PSL}(2, \mathbb{Z})$, in which a prototype of the harmonic volume appears. We define the harmonic volume. Let $\left(H^{\otimes 3}\right)^{\prime}$ be the kernel of the natural homomorphism $p: H^{\otimes 3} \ni a \otimes b \otimes c \mapsto$ $((a, b) c,(b, c) a,(c, a) b) \in H^{\oplus 3}$ induced by the intersection pairing on $H$. It is a subgroup of $K \otimes H$. The harmonic volume $I_{X}$ for $X$ is a restriction of the pointed harmonic volume $I_{(X, x)}$ :

$$
I_{X}=\left.I_{(X, x)}\right|_{\left(H^{\otimes 3}\right)^{\prime}}:\left(H^{\otimes 3}\right)^{\prime} \rightarrow \mathbb{R} / \mathbb{Z} .
$$

It depends only on the complex structure of $X$, and not on the choice of a Hermitian metric and base point. The mapping class group $\Gamma_{g}$ acts naturally on $H$. This induces the diagonal action of $\Gamma_{g}$ on $\operatorname{Hom}_{\mathbb{Z}}\left(\left(H^{\otimes 3}\right)^{\prime}, \mathbb{R} / \mathbb{Z}\right)$. Let Aut $X$ denote the group of biholomorphisms of $X$. By construction, $I_{X}$ is Aut $X$-invariant. It can be regarded as a real analytic section of a local system on $\mathcal{M}_{g}$ obtained by the $\Gamma_{g}$-module $\operatorname{Hom}_{\mathbb{Z}}\left(\left(H^{\otimes 3}\right)^{\prime}, \mathbb{R} / \mathbb{Z}\right)$. The harmonic volume $2 I_{X}$ for hyperelliptic curves is known to be trivial. Nevertheless, the zero locus of the harmonic volume for nonhyperelliptic curves is unknown. Moreover the harmonic volume $I_{X}$ can be interpreted as the volume of a 3 -chain in the torus $\mathbb{R}^{3} / \mathbb{Z}^{3}$. From this viewpoint, Faucette 9$]$ extended it to the higher-dimensional harmonic volume. See the end of Section 4

In Section 2.3, we introduce some basic concepts about algebraic cycles on an algebraic variety, in particular, the Jacobian variety $J(X)$ (or $J$ ) of $X$. Weil [36, pp. 331] asked whether homologically trivial algebraic cycles are algebraically nontrivial in $J$. That is, whether the Griffiths group is nontrivial, or roughly speaking, whether these algebraic cycles can be "continuously" (algebraically) deformed into the zero cycle. By the Abel-Jacobi map $X \rightarrow J$, $X$ is embedded in $J$. Its image of the $k$-th symmetric product of $X$ is denoted by $W_{k}$. The algebraic $k$-cycle $W_{k}-W_{k}^{-}$in $J$ is known to be homologous to zero. We denote by $W_{k}^{-}$the image of $W_{k}$ under multiplication by -1 . The cycle $W_{k}-W_{k}^{-}$is called the $k$-th Ceresa cycle for $1 \leq k \leq g-1$. It is one of the main subjects of this chapter. If $X$ is hyperelliptic or $k=g-1$, then the $k$-th Ceresa cycle is trivial. Ceresa [3] proved that the $k$-th Ceresa cycle is algebraically nontrivial in $J(X)$ for a generic compact Riemann surface $X$ of genus $g \geq 3$ for $1 \leq k \leq g-2$.

Section 3 establishes the relation between a higher Abel-Jacobi map and the harmonic volume. In preparation, we briefly sketch the Hodge structure of a $\mathbb{Z}$-module of finite rank. As the harmonic volume is the volume of a 3- 
chain in $\mathbb{R}^{3} / \mathbb{Z}^{3}$, it is regarded as a point of an intermediate Jacobian of $J$. Griffiths [1] defined the $k$-th Abel-Jacobi map $\Phi_{k}$ from the $k$-dimensional homologically trivial algebraic cycles on $J$ to the intermediate Jacobian of $J$ which is isomorphic to $\operatorname{Hom}_{\mathbb{Z}}\left(\wedge^{2 k+1} H, \mathbb{R} / \mathbb{Z}\right)$ here. By integration on a 3 -chain which bounds the first Ceresa cycle $W_{1}-W_{1}^{-}$, the harmonic volume $2 I_{X}$ can be identified with its image by the first Abel-Jacobi map of Griffiths, i.e.,

$$
2 I_{X}=\Phi_{1}\left(W_{1}-W_{1}^{-}\right) \in \operatorname{Hom}_{\mathbb{Z}}\left(\wedge^{3} H, \mathbb{R} / \mathbb{Z}\right) .
$$

This identification induces a sufficient condition for the nontriviality of the Ceresa cycle in $J$ by computing some values of $2 I_{X}$.

In Section 4, we review the pointed Torelli theorem as an application of the pointed harmonic volume. Hain [13] defined a mixed Hodge structure on the truncation of the fundamental group ring of complex manifolds by means of Chen's iterated integrals of 1-forms. This is a different approach to that of Morgan 22. The classical Torelli theorem says that a compact Riemann surface $X$ is determined by its Jacobian variety $J(X)$ regarded as a complex torus. Let $\pi_{1}(X, x)$ be the fundamental group of $X$ with base point $x$. We define the augmentation ideal $J_{x}$ of the group ring $\mathbb{Z} \pi_{1}(X, x)$ by the kernel of the map $\mathbb{Z} \pi_{1}(X, x) \ni \sum a_{\gamma} \gamma \mapsto \sum a_{\gamma} \in \mathbb{Z}$. The $k$-th power of the augmentation ideal is denoted by $J_{x}^{k}$. From Chen's $\pi_{1}$ de Rham theorem, see Section 2.1. the pointed harmonic volume belongs to $\operatorname{Hom}_{\mathbb{Z}}\left(J_{x} / J_{x}^{3}, \mathbb{R}\right)$. The pointed Torelli theorem states that the mixed Hodge structure on $\operatorname{Hom}_{\mathbb{Z}}\left(J_{x} / J_{x}^{3}, \mathbb{Z}\right)$ determines the structure of $\mathcal{C}_{g}$. Hain [13] and Pulte [28] gave a proof using the pointed harmonic volume and an extension obtained by a natural short exact sequence of mixed Hodge structures

$$
0 \longrightarrow H \longrightarrow \operatorname{Hom}_{\mathbb{Z}}\left(J_{x} / J_{x}^{3}, \mathbb{Z}\right) \longrightarrow K \longrightarrow 0 .
$$

In the proof of this theorem, the classical Torelli theorem follows from the preservation of extensions of the mixed Hodge structures. We obtain the biholomorphism of compact Riemann surfaces. When we distinguish the base points, the pointed harmonic volume plays an important role. Section 4 is devoted to this theorem.

In Section 5 , we deal with an application of the harmonic volume, the nontriviality of the Ceresa cycle $W_{k}-W_{k}^{-}$in the Jacobian variety $J(X)$. The harmonic volume captures information about how $X$ is embedded in $J$. From the nonvanishing of the first variation of the harmonic volume for special families of hyperelliptic curves, Harris [17] obtained a result similar to Ceresa's. The problem here is to give an explicitly compact Riemann surface satisfying the nontriviality condition. Harris [18] extended the harmonic volume with values in $\mathbb{C} / \mathbb{Z}[\sqrt{-1}]$ and proved that the first Ceresa cycle for the Fermat quartic $F_{4}$ of genus 3 is algebraically nontrivial in $J\left(F_{4}\right)$. Faucette [8] extended this and gave the proof that the second Ceresa cycle for an unramified double covering of $F_{4}$ is algebraically nontrivial. Bloch [1] further studied $F_{4}$ 
by means of $L$-functions. There are a few explicit nontrivial examples apart from this. In the following, we focus on nontriviality for the first Ceresa cycle. The author proves in 33 nontriviality for the Klein quartic $K_{4}$ of genus 3 by a slight modification of this method. The curve $K_{4}$ has a representation as a branched cyclic covering of $\mathbb{C} P^{1}$. From this, we can compute certain values of the harmonic volume for $K_{4}$ by special values of the generalized hypergeometric function ${ }_{3} F_{2}$. See Section 5.2 for ${ }_{3} F_{2}$. This method sheds some new light on nontriviality for the Ceresa cycles. Moreover, he proves in [34 nontriviality for the Fermat sextic $F_{6}$ of genus 10 using this method, but applying it directly to other Fermat curves is difficult. Let $\mathcal{O}$ denote the integer ring of the $N$-th cyclotomic field for a positive integer $N \geq 4$. Otsubo [26] extended the harmonic volume with values in $\left(\mathcal{O} \otimes_{\mathbb{Z}} \mathbb{R}\right) / \mathcal{O}$. He obtained its value and the algorithm in proving nontriviality for the $N$-th Fermat curves $F_{N}$ for any $N \geq 4$. See Sections 5.1 and 5.2. Moreover, he pointed out a relation between the harmonic volume and number theory using $L$-functions including nontorsionness of the higher Abel-Jacobi image $\Phi_{k}\left(W_{k}-W_{k}^{-}\right)$and the conjecture of Swinnerton-Dyer. Using his method, we 35] recently obtained nontriviality for some cyclic quotient $C_{N}$ of the Fermat curve. Here $N$ is a prime number with $N=1$ modulo 3. See Section 5.3. We remark that certain values of the harmonic volume for special Riemann surfaces were calculated, but all the values are unknown.

\section{Preliminaries}

\subsection{Iterated integrals}

To define the harmonic volume for a compact Riemann surface, we need to recall Chen's iterated integrals on a smooth manifold. They have been developed in various theories including the de Rham homotopy theory. We introduce iterated integrals of 1 -forms and $\pi_{1}$ de Rham theorem which states that the cohomology of the loop space of the smooth manifold can be calculated by means of these integrals. For a treatment of a more general case, we refer to Chen [6] and Hain [13.

Let $M$ be a smooth manifold and $\Omega^{i}(M)$ the smooth $i$-forms on $M$.

Definition 2.1. Denote $\gamma:[0,1] \rightarrow M$ as a piecewise smooth path. For $\omega_{1}, \omega_{2}, \ldots, \omega_{n} \in \Omega^{1}(M)$, we define an iterated (path) integral of $\omega_{1}, \omega_{2}, \ldots, \omega_{n}$ along $\gamma$ by

$$
\int_{\gamma} \omega_{1} \omega_{2} \cdots \omega_{n}=\int \cdots \int_{0 \leq t_{1} \leq t_{2} \leq \cdots \leq t_{n} \leq 1} f_{1}\left(t_{1}\right) f_{2}\left(t_{2}\right) \cdots f_{n}\left(t_{n}\right) d t_{1} d t_{2} \cdots d t_{n}
$$

where $f_{j}(t) d t$ is the pullback $\gamma^{*} \omega_{j}$. The number $n$ is called the length. 
The iterated integral is independent of the choice of the parameterization of the path $\gamma$. We introduce fundamental properties of iterated integrals. Let $P M$ be the set of piecewise smooth paths in $M$. For paths $\alpha, \beta \in P M$ such that $\alpha(1)=\beta(0)$, we denote their product by $\alpha \cdot \beta \in P M$ as usual. It is easy to prove

Lemma $2.2\left(\right.$ (4]). If $\omega_{1}, \omega_{2}, \ldots, \omega_{n} \in \Omega^{1}(M)$, then we have

$$
\int_{\alpha \cdot \beta} \omega_{1} \omega_{2} \cdots \omega_{n}=\sum_{i=0}^{r} \int_{\alpha} \omega_{1} \cdots \omega_{i} \int_{\beta} \omega_{i+1} \cdots \omega_{n}
$$

Here, we set $\int_{\gamma} \omega_{1} \omega_{2} \cdots \omega_{n}=1$ for $n=0$.

Examples 2.3.

$$
\begin{aligned}
\int_{\alpha \cdot \beta} \omega_{1} \omega_{2} & =\int_{\alpha} \omega_{1} \omega_{2}+\int_{\alpha} \omega_{1} \int_{\beta} \omega_{2}+\int_{\beta} \omega_{1} \omega_{2} \\
\int_{\alpha \cdot \beta \cdot \gamma} \omega_{1} \omega_{2} & =\left(\int_{\alpha}+\int_{\beta}+\int_{\gamma}\right) \omega_{1} \omega_{2}+\int_{\alpha} \omega_{1} \int_{\beta} \omega_{2}+\int_{\alpha} \omega_{1} \int_{\gamma} \omega_{2}+\int_{\beta} \omega_{1} \int_{\gamma} \omega_{2} .
\end{aligned}
$$

A permutation $\sigma$ of $\{1,2, \ldots, r+s\}$ is a shuffle of type $(r, s)$ if

$$
\sigma^{-1}(1)<\sigma^{-1}(2)<\cdots<\sigma^{-1}(r)
$$

and

$$
\sigma^{-1}(r+1)<\sigma^{-1}(r+2)<\cdots<\sigma^{-1}(r+s) .
$$

The following formula was derived by Ree [29].

Proposition 2.4 (Shuffle relation). Let $\omega_{1}, \omega_{2}, \ldots, \omega_{r+s} \in \Omega^{1}(M)$. Then we have

$$
\int_{\alpha} \omega_{1} \omega_{2} \cdots \omega_{r} \int_{\alpha} \omega_{r+1} \omega_{r+2} \cdots \omega_{r+s}=\sum_{\sigma} \int_{\alpha} \omega_{\sigma(1)} \omega_{\sigma(2)} \cdots \omega_{\sigma(r+s)},
$$

where $\sigma$ runs over the shuffles of type $(r, s)$.

\section{Examples 2.5.}

$$
\begin{aligned}
\int_{\alpha} \omega_{1} \int_{\alpha} \omega_{2} & =\int_{\alpha} \omega_{1} \omega_{2}+\int_{\alpha} \omega_{2} \omega_{1} \\
\int_{\alpha} \omega_{1} \int_{\alpha} \omega_{2} \omega_{3} & =\int_{\alpha} \omega_{1} \omega_{2} \omega_{3}+\int_{\alpha} \omega_{2} \omega_{3} \omega_{1}+\int_{\alpha} \omega_{2} \omega_{1} \omega_{3} .
\end{aligned}
$$

A function $F: P M \rightarrow \mathbb{R}^{m}$ is a homotopy functional if for every $\gamma \in P M$ $F(\gamma)$ depends only on the homotopy class of $\gamma$ relative to its endpoints. For 
each $x \in M$, a homotopy functional $F$ induces a function $\pi_{1}(M, x) \rightarrow \mathbb{R}$. It is well-known that a function

$$
\int \omega: P M \ni \gamma \mapsto \int_{\gamma} \omega \in \mathbb{R}
$$

is a homotopy functional if and only if $\omega \in \Omega^{1}(M)$ is closed. In general, even if $\omega_{1}, \omega_{2} \in \Omega^{1}(M)$ are closed, a function

$$
\int \omega_{1} \omega_{2}: P M \ni \gamma \mapsto \int_{\gamma} \omega_{1} \omega_{2} \in \mathbb{R}
$$

may not be a homotopy functional. For iterated integrals of length 2, we need a correction term $\eta \in \Omega^{1}(M)$.

Proposition 2.6. Let $\omega_{1}, \omega_{2}, \ldots, \omega_{r}, \eta \in \Omega^{1}(M)$ and $c_{i j} \in \mathbb{R}$ for $1 \leq i, j \leq r$. Suppose that each $\omega_{i}$ is closed. Then

$$
\sum c_{i j} \int \omega_{i} \omega_{j}+\int \eta
$$

is a homotopy functional if and only if

$$
d \eta+\sum c_{i j} \omega_{i} \wedge \omega_{j}=0
$$

This can be verified by lifting the integral to the universal covering of $M$. Let $B_{s}(M)$ be the vector space of finite linear combinations of iterated integrals on $M$ of length $\leq s$. Each elements of $B_{s}(M)$ is represented by

$$
I=\lambda+\sum a_{i} \int \omega_{i}+\sum a_{i j} \int \omega_{i} \omega_{j}+\cdots+\sum_{|J|=s} a_{J} \int \omega_{j_{1}} \omega_{j_{2}} \cdots \omega_{j_{s}} .
$$

For the group ring $\mathbb{Z} \pi_{1}(X, x)$, we define the augmentation ideal $J_{x}$ by the kernel of the map $\mathbb{Z} \pi_{1}(X, x) \ni \sum a_{\gamma} \gamma \mapsto \sum a_{\gamma} \in \mathbb{Z}$. It is clear that $J_{x}$ is generated by $\gamma-1$ for any loop $\gamma$ with base point $x$. Here 1 denotes the constant path at $x$. The $k$-th power of the augmentation ideal is denoted by $J_{x}^{k}$. For $I=\int \omega \in B_{1}(M)$ and $(\alpha-1)(\beta-1) \in J_{x}^{2}$, we have immediately

$$
\langle I,(\alpha-1)(\beta-1)\rangle=0,
$$

where $\alpha, \beta$ are loops with base point $x$. Moreover, Examples 2.3 imply

$$
\langle I,(\alpha-1)(\beta-1)(\gamma-1)\rangle=0
$$

for $I \in B_{2}(M)$ and $(\alpha-1)(\beta-1)(\gamma-1) \in J_{x}^{3}$. We generalize these expressions.

Lemma 2.7. Let $\alpha_{1}, \alpha_{2}, \ldots, \alpha_{s}$ denote loops with base point $x$. If $I \in B_{r}(M)$ and $r<s$, then we have

$$
\left\langle I,\left(\alpha_{1}-1\right)\left(\alpha_{2}-1\right) \cdots\left(\alpha_{s}-1\right)\right\rangle=0 .
$$


The proof of this lemma is straightforward. Let $\omega_{1}, \omega_{2}, \ldots, \omega_{s} \in \Omega^{1}(M)$ and $\left(\alpha_{1}-1\right)\left(\alpha_{2}-1\right) \cdots\left(\alpha_{s}-1\right)$ as above. Lemma 2.2 implies

$$
\left\langle\int \omega_{1} \omega_{2} \cdots \omega_{s},\left(\alpha_{1}-1\right)\left(\alpha_{2}-1\right) \cdots\left(\alpha_{s}-1\right)\right\rangle=\int_{\alpha_{1}} \omega_{1} \int_{\alpha_{2}} \omega_{2} \cdots \int_{\alpha_{s}} \omega_{s} .
$$

The set of piecewise smooth loops in $M$ with base point $x \in M$ is denoted by $P_{x} M$. Let $H^{0}\left(B_{s}(M), x\right)$ be the set of homotopy functionals $P_{x} M \rightarrow \mathbb{R}$. Integration induces a linear map

$$
H^{0}\left(B_{s}(M), x\right) \ni I \mapsto(\gamma \mapsto\langle I, \gamma\rangle) \in \operatorname{Hom}_{\mathbb{Z}}\left(\mathbb{Z} \pi_{1}(X, x), \mathbb{R}\right) .
$$

Lemma 2.7 gives us $\left.I\right|_{J_{x}^{s+1}}=0$ for $I \in B_{s}(M)$. Chen proved the following $\pi_{1}$ de Rham theorem [5, Theorem 5.3].

Theorem 2.8. The integration map induces an isomorphism

$$
H^{0}\left(B_{s}(M), x\right) \rightarrow \operatorname{Hom}_{\mathbb{Z}}\left(\mathbb{Z} \pi_{1}(X, x) / J_{x}^{s+1}, \mathbb{R}\right) .
$$

Let $\bar{B}_{s}(M)$ denote the subset of elements of $B_{s}(M)$ whose constant term vanishes. A similar result follows.

Corollary 2.9. We have an isomorphism

$$
H^{0}\left(\bar{B}_{s}(M), x\right) \rightarrow \operatorname{Hom}_{\mathbb{Z}}\left(J_{x} / J_{x}^{s+1}, \mathbb{R}\right) .
$$

If we replace $\mathbb{R}$ with $\mathbb{C}$, similar results are obtained. The pointed harmonic volume $I_{(X, x)}$ for a pointed compact Riemann surface $(X, x)$ can be interpreted as an element of $H^{0}\left(\bar{B}_{2}(X), x\right)$.

\subsection{Harmonic volume}

We review the definition of the harmonic volume for a compact Riemann surface and its properties. It is a complex analytic invariant defined by Chen's iterated integrals of length 2 . It also gives information about how the compact Riemann surface is embedded in its Jacobian variety. First, we define the pointed harmonic volume for a pointed compact Riemann surface.

Let $X$ be a compact Riemann surface or smooth projective curve over $\mathbb{C}$ of genus $g \geq 2$. (See Farkas and Kra [7] for an introduction to Riemann surfaces.) The surface $X$ is homeomorphic to an oriented closed surface $\Sigma_{g}$ of genus $g$. Its mapping class group, denoted by $\Gamma_{g}$, is the group of isotopy classes of orientation-preserving diffeomorphisms of $\Sigma_{g}$. The group $\Gamma_{g}$ acts naturally on the first integral homology group $H_{1}(X ; \mathbb{Z})=H_{1}\left(\Sigma_{g} ; \mathbb{Z}\right)$. Let $H$ denote the first integral cohomology group $H^{1}(X ; \mathbb{Z})$. By Poincaré duality, $H$ is isomorphic to $H_{1}(X ; \mathbb{Z})$ as $\Gamma_{g}$-modules. The Hodge star operator $*$ is 
locally given by $*\left(f_{1}(z) d z+f_{2}(z) d \bar{z}\right)=-\sqrt{-1} f_{1}(z) d z+\sqrt{-1} f_{2}(z) d \bar{z}$ in a local coordinate $z$. It depends only on the complex structure and not on the choice of a Hermitian metric. The real Hodge star operator $*: \Omega^{1}(X) \rightarrow \Omega^{1}(X)$ is given by restriction. Using the Hodge theorem, we identify $H$ with the space of real harmonic 1-forms on $X$ with $\mathbb{Z}$-periods, i.e., $H=\left\{\omega \in \Omega^{1}(X) ; d \omega=\right.$ $d * \omega=0, \int_{\gamma} \omega \in \mathbb{Z}$ for any loop $\left.\gamma\right\}$. We introduce the following lemma for homotopy functionals. See Proposition 2.6.

Lemma 2.10. Let $\omega_{i} \in \Omega^{1}(X)$ and $c_{i j} \in \mathbb{R}$. If $\sum \int_{X} c_{i j} \omega_{i} \wedge \omega_{j}=0$, then there exists a 1-form $\eta \in \Omega^{1}(X)$ such that $d \eta+\sum c_{i j} \omega_{i} \wedge \omega_{j}=0$.

Indeed, an exact sequence

$$
0 \longrightarrow \mathbb{C} \longrightarrow \Omega^{0}(X) \stackrel{d * d}{\longrightarrow} \Omega^{2}(X) \stackrel{\int_{X}}{\longrightarrow} \mathbb{C} \longrightarrow 0
$$

gives a function $h \in \Omega^{0}(X)$ such that $d * d h=\sum c_{i j} \omega_{i} \wedge \omega_{j}$. Here the left vector space $\mathbb{C}$ means the constant functions. Put $\eta=-* d h$.

Let $x \in X$ be a point. We define the pointed harmonic volume for $(X, x)$ in the following way. Let $K$ be the kernel of $():, H \otimes H \rightarrow \mathbb{Z}$ induced by the intersection pairing. For a given $\sum_{i=1}^{n} a_{i} \otimes b_{i} \in K$, there exists an $\eta \in \Omega^{1}(X)$ satisfying the two conditions

$$
d \eta+\sum_{i=1}^{n} a_{i} \wedge b_{i}=0
$$

and

$$
\int_{X} \eta \wedge * \alpha=0
$$

for any closed 1-form $\alpha \in \Omega^{1}(X)$. The second condition determines $\eta$ uniquely. We can choose $\eta=-* d h$ with $d * d h=\sum_{i=1}^{n} a_{i} \wedge b_{i}$ as in the above lemma. This $\eta$ readily satisfies the above two conditions. For any pointed compact Riemann surface $(X, x)$, the homotopy functional

$$
\varphi: P_{x} X \ni \gamma \mapsto \sum_{i=1}^{n} \int_{\gamma} a_{i} b_{i}+\int_{\gamma} \eta \in \mathbb{R}
$$

induces a map

$$
\bar{\varphi}: \mathbb{Z} \pi_{1}(X, x) \rightarrow \mathbb{R}
$$

We remark that this $\bar{\varphi}$ is an element of $H^{0}\left(\bar{B}_{2}(X), x\right) \cong \operatorname{Hom}_{\mathbb{Z}}\left(J_{x} / J_{x}^{3}, \mathbb{R}\right)$. Lemma 2.2 yields

$$
\varphi(\alpha \beta)=\varphi(\alpha)+\varphi(\beta)+\sum_{i=1}^{n} \int_{\alpha} a_{i} \int_{\beta} b_{i}
$$


for loops $\alpha, \beta \in P_{x} X$. From the assumption, $\int_{\alpha} a_{i}, \int_{\beta} b_{i} \in \mathbb{Z}$ for each $i$. Using a natural projection $\mathbb{R} \rightarrow \mathbb{R} / \mathbb{Z}$, the map $\bar{\varphi}: \mathbb{Z} \pi_{1}(X, x) \rightarrow \mathbb{R} / \mathbb{Z}$ is a homomorphism. Furthermore, this gives the homomorphism

$$
\bar{\varphi}: H_{1}(X ; \mathbb{Z}) \rightarrow \mathbb{R} / \mathbb{Z}
$$

We define the notion of pointed harmonic volume [28].

Definition 2.11. For $\sum_{i=1}^{n} a_{i} \otimes b_{i} \in K$ and $c \in H$, the pointed harmonic volume is a homomorphism $K \otimes H \rightarrow \mathbb{R} / \mathbb{Z}$

$$
I_{(X, x)}\left(\left(\sum_{i=1}^{n} a_{i} \otimes b_{i}\right) \otimes c\right)=\bar{\varphi}(c) \quad \bmod \mathbb{Z} .
$$

Here $\bar{\varphi}$ is defined in the way stated above and the homology class $c$ is considered as a loop in $X$ with base point $x$.

Remark 2.12. From Proposition 2.4, we have

$$
I_{(X, x)}\left(\left(\sum_{i=1}^{n} a_{i} \otimes b_{i}\right) \otimes c\right)=-I_{(X, x)}\left(\left(\sum_{i=1}^{n} b_{i} \otimes a_{i}\right) \otimes c\right) \quad \bmod \mathbb{Z} .
$$

Harris [17] gave the same definition of $I_{(X, x)}$, and called the pointed harmonic volume by Pulte. The pointed harmonic volume $I_{(X, x)}$ is naturally regarded as an element of $\operatorname{Hom}_{\mathbb{Z}}\left(\wedge^{3} H, \mathbb{R} / \mathbb{Z}\right)$ and a section of the local system $\mathcal{L}_{1}$ on $\mathcal{C}_{g}$ defined in Section 1 .

The harmonic volume is a restriction of the pointed harmonic volume $I_{(X, x)}$. We denote by $\left(H^{\otimes 3}\right)^{\prime}$ the kernel of the natural homomorphism $p: H^{\otimes 3} \rightarrow H^{\oplus 3}$ defined by $p(a \otimes b \otimes c)=((a, b) c,(b, c) a,(c, a) b)$. The group $K \otimes H$ is a subgroup of $\left(H^{\otimes 3}\right)^{\prime}$. We have a natural short exact sequence

$$
0 \longrightarrow\left(H^{\otimes 3}\right)^{\prime} \longrightarrow H^{\otimes 3} \stackrel{p}{\longrightarrow} H^{\oplus 3} \longrightarrow 0 .
$$

The rank of the free $\mathbb{Z}$-module $\left(H^{\otimes 3}\right)^{\prime}$ is $(2 g)^{3}-6 g$.

Definition 2.13 (17]). The harmonic volume $I_{X}$ for $X$ is a linear form on $\left(H^{\otimes 3}\right)^{\prime}$ with values in $\mathbb{R} / \mathbb{Z}$ defined by the restriction of $I_{(X, x)}$ to $\left(H^{\otimes 3}\right)^{\prime}$, i.e.,

$$
I_{X}=\left.I_{(X, x)}\right|_{\left(H^{\otimes 3}\right)^{\prime}}:\left(H^{\otimes 3}\right)^{\prime} \rightarrow \mathbb{R} / \mathbb{Z} .
$$

From Lemma 2.2, the harmonic volume $I_{X}$ is independent of the choice of base point $x$. Let $S_{3}$ be the third symmetric group. We explain a cyclic invariance of $I_{X}$ by the natural action of $S_{3}$ on $\left(H^{\otimes 3}\right)^{\prime}$. Combining Stokes' 
theorem and Remark 2.12, we have

$I_{X}\left(\sum_{i} \omega_{\sigma(1), i} \otimes \omega_{\sigma(2), i} \otimes \omega_{\sigma(3), i}\right)=\operatorname{sgn}(\sigma) I_{X}\left(\sum_{i} \omega_{1, i} \otimes \omega_{2, i} \otimes \omega_{3, i}\right) \bmod \mathbb{Z}$,

where $\sum_{i} \omega_{1, i} \otimes \omega_{2, i} \otimes \omega_{3, i} \in\left(H^{\otimes 3}\right)^{\prime}$ and $\sigma$ is an element of $S_{3}$.

We present examples of calculation of the harmonic volume for hyperelliptic curves. The hyperelliptic curve $C$ is the compactification of the plane curve in the $(z, w)$ plane $\mathbb{C}^{2}$

$$
w^{2}=\prod_{i=0}^{2 g+1}\left(z-p_{i}\right)
$$

where $p_{0}, p_{1}, \ldots, p_{2 g+1}$ are some distinct points on $\mathbb{C}$. It admits the hyperelliptic involution given by $(z, w) \mapsto(z,-w)$. Let $\pi$ be the 2 -sheeted covering $C \rightarrow \mathbb{C} P^{1},(z, w) \mapsto z$, branched over $2 g+2$ branch points $\left\{p_{i}\right\}_{i=0,1, \cdots, 2 g+1}$ and $P_{i} \in C$ a ramification point such that $\pi\left(P_{i}\right)=p_{i}$. It is known that $\left\{P_{i}\right\}_{i=0,1, \ldots, 2 g+1}$ is just the set of all the Weierstrass points on any hyperelliptic curve $C$. We outline the computation of the harmonic volumes for hyperelliptic curves. See [31, 32 for details. For any hyperelliptic curve $C$, one has $I_{C}=0$ or $1 / 2 \bmod \mathbb{Z}$ by the existence of the hyperelliptic involution. The computation was performed using a suitable choice of symplectic basis $\left\{x_{i}, y_{i}\right\}_{i=1, \ldots, g}$ of $H([31$, p.800]). For example,

$$
I_{C}\left(\left(x_{i} \otimes y_{i}-x_{k+1} \otimes y_{k+1}\right) \otimes y_{k}\right)=\left\{\begin{array}{cc}
\frac{1}{2} & (i<k, 2 \leq k \leq g-1) \\
0 & \text { otherwise }
\end{array}\right.
$$

We have two ways to compute the harmonic volumes for all the hyperelliptic curves. First the computation can be reduced to that of a single hyperelliptic curve. Second we use basic results from the cohomology group of the hyperelliptic mapping class group which is composed of the centralizer of the hyperelliptic involution in $\Gamma_{g}$. Similarly the author [32] obtained the pointed harmonic volume $I_{\left(C, P_{j}\right)}$ for the Weierstrass pointed hyperelliptic curves. However, the pointed harmonic volume for other pointed hyperelliptic curves has yet to be determined.

We may consider the harmonic volume as an element of $\operatorname{Hom}_{\mathbb{Z}}\left(\left(\wedge^{3} H\right)^{\prime}, \mathbb{R} / \mathbb{Z}\right)$. Let $j_{2}: H^{\otimes 3} \rightarrow \wedge^{3} H$ be a natural homomorphism

$$
j_{2}(a \otimes b \otimes c)=a \wedge b \wedge c
$$


where $\wedge^{3} H$ denotes the third exterior product of $H$. We have a homomorphism of short exact sequences

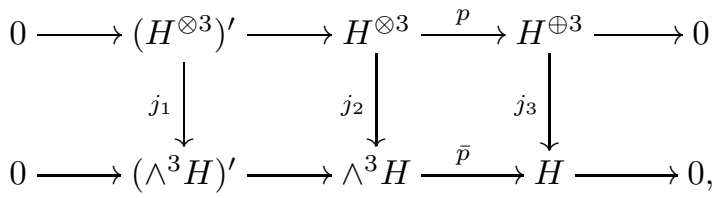

where $j_{3}(a, b, c)=a+b+c, \bar{p}(a \wedge b \wedge c)=(a, b) c+(b, c) a+(c, a) b$ and $j_{1}$ is the restriction homomorphism of $j_{2}$ to $\left(H^{\otimes 3}\right)^{\prime}$. The rank of the free $\mathbb{Z}$-module $\left(\wedge^{3} H\right)^{\prime}$ is $\left(\begin{array}{c}2 g \\ 3\end{array}\right)-2 g$. Using Formula (2.3), it is easy to show the following:

Proposition 2.14. We can take a homomorphism $\nu_{X} \in \operatorname{Hom}_{\mathbb{Z}}\left(\left(\wedge^{3} H\right)^{\prime}, \mathbb{R} / \mathbb{Z}\right)$ satisfying the commutative diagram

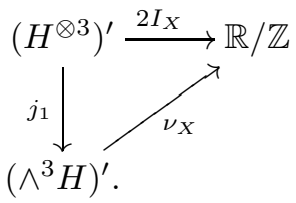

The homomorphism $\nu_{X}$ is also called the harmonic volume. Another description of $\nu_{X}$ is as follows. For simplicity, we consider $\omega_{1} \wedge \omega_{2} \wedge \omega_{3} \in\left(\wedge^{3} H\right)^{\prime}$, and fix a base point $x \in X$. The map $A_{1}: X \rightarrow \mathbb{R}^{3} / \mathbb{Z}^{3}$, a kind of Abel-Jacobi map, is defined by:

$$
A_{1}(y)=\left(\int_{x}^{y} \omega_{1}, \int_{x}^{y} \omega_{2}, \int_{x}^{y} \omega_{3}\right) \bmod \mathbb{Z}^{3} .
$$

There exists a 3 -chain $c_{3}$ in $\mathbb{R}^{3} / \mathbb{Z}^{3}$ such that the image $A_{1}(X)=\partial c_{3}$ modulo integral 2-chains. For each $k=1,2,3$, we can take the coordinates $x_{k}$ on $\mathbb{R}^{3} / \mathbb{Z}^{3}$ such that $A_{1}^{*}\left(d x_{k}\right)=\omega_{k}$. The volume of $c_{3}$ modulo $\mathbb{Z}$ is independent of the choice of $c_{3}$ and base point $x$. The following proposition is proved by a straightforward computation.

Proposition 2.15. We have

$$
\nu_{X}\left(\omega_{1} \wedge \omega_{2} \wedge \omega_{3}\right)=2 \int_{c_{3}} d x_{1} \wedge d x_{2} \wedge d x_{3} \quad \bmod \mathbb{Z}
$$

\subsection{The Chow group}

The harmonic volume can be applied to the nontriviality of the Ceresa cycles in the Jacobian varieties of compact Riemann surfaces. From this complex analytic method, the harmonic volume can capture more detailed information 
of these cycles. The Ceresa cycle is an element of the Chow group that, to begin, we need to define. A general reference here is Fulton 10.

Let $V$ be a smooth projective variety over $\mathbb{C}$. The group $\mathcal{Z}_{k}(V)$ is defined to be the free abelian group generated by the irreducible subvarieties $W$ on $V$ of dimension $k$. It is called the group of algebraic cycles of dimension $k$. We consider the algebraic equivalence on $V$. The algebraic cycle $Z \in \mathcal{Z}_{k}(V)$ is algebraically equivalent to zero if there exists a smooth curve $C$, a cycle $T \in \mathcal{Z}_{k}(V \times C)$, and two points $x_{1}, x_{2} \in C$ such that $Z=i_{1}^{*}(T)-i_{2}^{*}(T)$, where $i_{j}: V \hookrightarrow V \times C$ is $i_{j}(x)=\left(x, x_{j}\right)$ for $j=1,2$. For $C=\mathbb{C} P^{1}$, we call it rationally equivalent. Denote

$$
\mathcal{Z}_{k}(V) \text { alg }=\left\{Z \in \mathcal{Z}_{k}(V) ; Z \text { is algebraically equivalent to } 0\right\}
$$

and $\mathcal{Z}_{k}(V)_{\text {rat }}$ in the similar way. We define the Chow group of dimension $k$ by

$$
\mathrm{CH}_{k}(V):=\mathcal{Z}_{k}(V) / \mathcal{Z}_{k}(V)_{\text {rat }} \text {. }
$$

Set $\mathrm{CH}_{k}(V)_{\text {alg }}:=\mathcal{Z}_{k}(V)_{\text {alg }} / \mathcal{Z}_{k}(V)_{\text {rat }}$ which is the group of algebraically trivial cycles modulo rational equivalence. The cycle class map $\mathrm{CH}_{k}(V) \rightarrow H_{2 k}(V ; \mathbb{Z})$ is obtained by linearly extending the map to a subvariety $i$ : $W \hookrightarrow V$ associated with its homology class $i_{*}[W]$. The kernel of this map is denoted by $\mathrm{CH}_{k}(V)_{\text {hom. }}$. It is the group of homologically trivial cycles modulo rational equivalence. We have known inclusions

$$
\mathrm{CH}_{k}(V) \text { alg } \subset \mathrm{CH}_{k}(V)_{\text {hom }} \subset \mathrm{CH}_{k}(V) \text {. }
$$

The problem is to determine whether a cycle homologous to zero in $V$ is algebraically equivalent to zero. If the cycle homologous to zero is not, then we find the Griffiths group of $V$ is nontrivial. Here the $k$-th Griffith group $\operatorname{Griff}_{k}(V)$ is defined by $\operatorname{Griff}_{k}(V):=\mathrm{CH}_{k}(V)_{\text {hom }} / \mathrm{CH}_{k}(V)_{\text {alg. In particular, }}$ we are interested in this group for varieties and cycles defined over $\mathbb{Z}$. See [27] for example.

In this subsection and Sections 3 and 4 , we use the notation $H^{1}=H^{1}(X ; \mathbb{Z})$ and $H_{1}=H_{1}(X ; \mathbb{Z})$. Let $J(X)$ (or $\left.J\right)$ be the Jacobian variety of the compact Riemann surface $X$ of genus $g \geq 3$. We denote by $H^{1,0}$ the complex vector space of holomorphic 1 -forms on $X$, and fix a base point $x \in X$. The AbelJacobi map $X \rightarrow J$ is defined by

$$
A_{x}: X \ni y \mapsto\left(\omega \mapsto \int_{x}^{y} \omega\right) \in J=\left(H^{1,0}\right)^{\vee} / H_{1},
$$

where ${ }^{\vee}$ means the complex linear dual. We define an inclusion map by $H_{1} \ni$ $\gamma \mapsto\left(\omega \mapsto \int_{\gamma} \omega\right) \in\left(H^{1,0}\right)^{\vee}$. If we fix $\omega_{1}, \omega_{2}, \ldots, \omega_{g}$ as a basis of $H^{1,0}$, then $J$ is a $g$-dimensional complex torus obtained as the quotient of $\mathbb{C}^{g}$ by an abelian group. Since $g$ is positive, $X$ can be embedded into $J$. We may identify $H^{1}$ with $H^{1}(J ; \mathbb{Z})$ and $\wedge^{k} H^{1}$ with $H^{k}(J ; \mathbb{Z})$ for $1 \leq k \leq 2 g$. Let $X^{k}$ denote the $k$-fold product of $X$ and let $X_{k}$ denote the $k$-th symmetric product. We have 
a commutative diagram

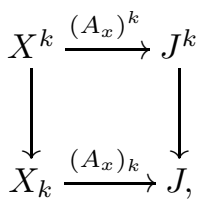

where the left-hand side map is the natural projection, the right-hand an addition, and $\left(A_{x}\right)_{k}$ is the induced homomorphism. With an abuse of notation, we also denote the latter by $A_{x}$; its image is denoted by $W_{k}(x):=A_{x}\left(X_{k}\right)$. For $x, y \in X$, the algebraic $k$-cycle $W_{k}(x)-W_{k}^{ \pm}(y)$ in $J$ is called the $k$-th Ceresa cycle. Here we denote by $W_{k}^{-}(y)$ the image of $W_{k}(y)$ under multiplication by -1 and $W_{k}^{+}(y)=W_{k}(y)$. The multiplication induces the identity map on $H_{2 k}(J ; \mathbb{Z})$. The $k$-th Ceresa cycle is homologous to zero, i.e., $W_{k}(x)-W_{k}^{ \pm}(y) \in$ $\mathrm{CH}_{k}(J)_{\text {hom }}$. Since $W_{k}(x)-W_{k}(y) \in \mathrm{CH}_{k}(J)_{\text {alg }}$, the class of $W_{k}(x)-W_{k}^{-}(y)$ modulo algebraic equivalence does not depend on $x$ and $y$. We omit the base points $x, y$, unless otherwise stated.

If $X$ is hyperelliptic, then $W_{k}-W_{k}^{-} \in \mathrm{CH}_{k}(J)_{\text {alg. Indeed, we may choose }}$ a Weierstrass point $x \in X$ as base point. The hyperelliptic curve $X$ has the hyperelliptic involution $\iota$ which is a biholomorphism of $X$ of order 2 and fixes all the Weierstrass points in $X$. Since the action of $\iota$ on $H_{1}$ is multiplication by -1 , the multiplication by -1 on $J(X)$ restricts to $\iota$ on $X$. Then we have $W_{k}-W_{k}^{-} \in \mathrm{CH}_{k}(J)_{\text {alg. }}$. If $k=g-1$, then the cycle $W_{g-1}-W_{g-1}^{-}$is known to be trivial. Ceresa's theorem [3] implies that $\operatorname{Griff}_{k}(J(X)) \neq 0$ for a generic (nonhyperelliptic) curve $X$ of genus $g \geq 3$ for $1 \leq k \leq g-2$. In Section 5, we give explicit $X$ 's such that $\operatorname{Griff}_{k}(J(X)) \neq 0$.

\section{Abel-Jacobi maps and harmonic volume}

To show a relation between the harmonic volume $\nu_{X}=2 I_{X}$ and the image of the Abel-Jacobi map of Griffiths, we begin to recall the definition of the

Hodge structure on a $\mathbb{Z}$-module of finite rank and an intermediate Jacobian of $V$. We give a sufficient condition for the Ceresa cycle of $\mathrm{CH}_{1}(J(X))_{\text {hom }}$ to be algebraically nontrivial.

A Hodge structure of weight- $w$ on a $\mathbb{Z}$-module $H_{\mathbb{Z}}$ of finite rank is a direct sum decomposition

$$
H_{\mathbb{C}}:=H_{\mathbb{Z}} \otimes_{\mathbb{Z}} \mathbb{C}=\bigoplus_{p+q=w} H^{p, q} \text { with } H^{p, q}=\overline{H^{q, p}} .
$$

The Hodge filtration associated to this Hodge structure is given by

$$
F^{p} H_{\mathbb{C}}=\bigoplus_{r \geq p} H^{r, s} .
$$


We immediately obtain $H^{p, q}=F^{p} H_{\mathbb{C}} \cap \overline{F^{q} H_{\mathbb{C}}}$ and the decreasing filtration

$$
H_{\mathbb{C}} \supset \cdots \supset F^{p} H_{\mathbb{C}} \supset F^{p+1} H_{\mathbb{C}} \supset \cdots .
$$

Conversely, a decreasing filtration $F^{p}$ of $H_{\mathbb{C}}$ with the condition $F^{p} \cap \overline{F^{q}}=0$ whenever $p+q=k+1$ determines a weight- $k$ Hodge structure by putting

$$
H^{p, q}=F^{p} \cap \overline{F^{q}} .
$$

If $V$ is a compact Kähler manifold, the cohomology group $H^{w}(V ; \mathbb{Z})$ underlies a Hodge structure of weight- $w$. Here, $H^{p, q}$ is the space of cohomology classes whose harmonic representative is of type $(p, q)$. For two Hodge structures $A$ and $B$ of weight $w$ and $v$ respectively, we have Hodge structures $A \otimes B$ and $\operatorname{Hom}(A, B)$ of weight $w+v$ and $v-w$ respectively:

$$
(A \otimes B)^{p, q}=\bigoplus_{i, j} A^{i, j} \otimes B^{p-i, q-j}
$$

and

$$
\operatorname{Hom}(A, B)^{p, q}=\left\{f: A_{\mathbb{C}} \rightarrow B_{\mathbb{C}} ; f\left(A^{i, j}\right) \subset B^{i+p, j+q}\right\} .
$$

Here $A_{\mathbb{C}}=\oplus_{p+q=w} A^{p, q}$ and $B_{\mathbb{C}}=\oplus_{p+q=v} B^{p, q}$. If $V$ is a compact Kähler manifold, the homology group $H_{w}(V ; \mathbb{Z})$ carries a natural Hodge structure of weight $-k$. Indeed, we recall the isomorphism $H_{k}(V ; \mathbb{C}) \cong \operatorname{Hom}_{\mathbb{C}}\left(H^{k}(V ; \mathbb{C}), \mathbb{C}\right)$ and obtain the direct sum decomposition

$$
H_{w}(V ; \mathbb{C})=\bigoplus_{-p-q=-w} H_{w}(V ; \mathbb{C})^{-p,-q},
$$

where $H_{w}(V ; \mathbb{C})^{-p,-q}=\left\{f: H^{w}(V ; \mathbb{C}) \rightarrow \mathbb{C} ; f\left(H^{r, s}\right)=0\right.$ whenever $(r, s) \neq$ $(p, q)\}$.

If $H_{\mathbb{Z}}$ has a Hodge structure of odd weight $2 k+1$, we define a complex torus by

$$
J(H):=H_{\mathbb{C}} /\left(F^{k+1} H_{\mathbb{C}}+H_{\mathbb{Z}}\right)=\overline{F^{k+1} H_{\mathbb{C}}} / H_{\mathbb{Z}}
$$

The real torus $H_{\mathbb{R}} / H_{\mathbb{Z}}$ is denoted by $J_{\mathbb{R}} H$, where $H_{\mathbb{R}}$ denotes $H_{\mathbb{Z}} \otimes_{\mathbb{Z}} \mathbb{R}$. The inclusion $H_{\mathbb{R}} \rightarrow H_{\mathbb{C}}$ induces an isomorphism of real Lie groups $J_{\mathbb{R}} H \rightarrow J(H)$. A natural projection $\mathbb{R} \rightarrow \mathbb{R} / \mathbb{Z}$ induces an isomorphism of real tori

$$
J_{\mathbb{R}} \operatorname{Hom}(H, \mathbb{C}) \cong \operatorname{Hom}_{\mathbb{Z}}\left(H_{\mathbb{Z}}, \mathbb{R} / \mathbb{Z}\right) .
$$

Lemma 3.1. We have a natural isomorphism of real tori

$$
J \operatorname{Hom}(H, \mathbb{C}) \rightarrow \operatorname{Hom}_{\mathbb{Z}}\left(H_{\mathbb{Z}}, \mathbb{R} / \mathbb{Z}\right) .
$$


We focus on the homology group $H_{2 k+1}(V ; \mathbb{Z})$ with Hodge structure of weight $-2 k-1$. The $k$-th intermediate Jacobian of Griffiths is defined by

$$
J_{k}(V):=J\left(H_{2 k+1}(V ; \mathbb{Z})\right)=\frac{F^{-k} H_{2 k+1}(V ; \mathbb{C})}{H_{2 k+1}(V ; \mathbb{Z})}=\frac{\left(F^{k+1} H^{2 k+1}(V ; \mathbb{C})\right)^{\vee}}{H_{2 k+1}(V ; \mathbb{Z})},
$$

The $J_{0}(X)$ is the Jacobian variety $J=J(X)$.

For an element $Z \in \mathrm{CH}_{k}(V)_{\text {hom }}$, we can take a topological $(2 k+1)$-chain $W$ so that $Z=\partial W$. The integration $H^{2 k+1}(V ; \mathbb{C}) \ni \omega \mapsto \int_{W} \omega \in \mathbb{C}$ induces the Abel-Jacobi map of Griffiths

$$
\Phi_{k}: \mathrm{CH}_{k}(V)_{\mathrm{hom}} \rightarrow J_{k}(V),
$$

where $\omega$ is a harmonic $(2 k+1)$-form on $V$ with integral periods 28, Section 4]. We give a key lemma in proving nontriviality for the Ceresa cycles.

Lemma 3.2. Let $F^{p}$ denote $F^{p} H^{2 k+1}(V ; \mathbb{C})$. If the image $\Phi_{k}(Z)$ is nonvanishing on $F^{k+2}+\overline{F^{k+2}} \subset H^{2 k+1}(V ; \mathbb{C})$ for $Z \in \mathrm{CH}_{k}(V)_{h o m}$, then $Z$ is not an element of $\mathrm{CH}_{k}(V)_{\text {alg }}$.

Proof. We have only to prove that the image of $\Phi_{k}$ on $\mathrm{CH}_{k}(V)$ alg vanishes on $F^{k+2}+\overline{F^{k+2}} \subset H^{2 k+1}(V ; \mathbb{C})$. If an algebraic cycle $Z$ is algebraically equivalent to zero in $V$, then there exists a topological $(2 k+1)$-chain $W$ such that $\partial W=Z$ and $W$ lies on $S$, where $S$ is an algebraic (or complex analytic) subset of $V$ of complex dimension $k+1$. The chain $W$ is unique up to $(2 k+1)$-cycles. We may assume that $\omega$ consists of the elements of $H^{p, q}=F^{p} \cap \overline{F^{q}}$ for $p>k+1$ or $q>k+1$. Then we have $\int_{W} \omega=0$.

From Lemma 3.1, we have $J_{k}(V) \cong \operatorname{Hom}_{\mathbb{Z}}\left(H^{2 k+1}(V ; \mathbb{Z}), \mathbb{R} / \mathbb{Z}\right)$. Suppose that $V$ is the Jacobian variety $J=J(X)$. Using the identification $H^{2 k+1}(J ; \mathbb{Z})=$ $\wedge^{2 k+1} H^{1}$, we may consider the Abel-Jacobi map of Griffiths as the homomorphism

$$
\Phi_{k}: \mathrm{CH}_{k}(J)_{\mathrm{hom}} \rightarrow \operatorname{Hom}\left(\wedge^{2 k+1} H^{1}, \mathbb{R} / \mathbb{Z}\right) .
$$

We consider $k=1$ in this subsection. Let $\nu^{*}$ denote the Abel-Jacobi image $\Phi_{1}\left(W_{1}-W_{1}^{-}\right)$. Harris ([17, 19, Proposition 2.1]) proved that $\left(\wedge^{3} H^{1}\right)^{\prime}$ can be identified with the primitive subgroup of $H^{3}(J ; \mathbb{Z})=\wedge^{3} H^{1}$ in the sense of Lefschetz, denoted by $H_{\text {prim }}^{3}(J ; \mathbb{Z})$. By this identification and the natural projection $\operatorname{Hom}_{\mathbb{Z}}\left(H^{3}(J ; \mathbb{Z}), \mathbb{R} / \mathbb{Z}\right) \rightarrow \operatorname{Hom}_{\mathbb{Z}}\left(H_{\text {prim }}^{3}(J ; \mathbb{Z}), \mathbb{R} / \mathbb{Z}\right)$, we consider $\nu^{*}$ as an element of $\operatorname{Hom}_{\mathbb{Z}}\left(\left(\wedge^{3} H^{1}\right)^{\prime}, \mathbb{R} / \mathbb{Z}\right)$. From Proposition 2.15, the 3-cycle $c_{3}$ in the torus $\mathbb{R}^{3} / \mathbb{Z}^{3}$ can be identified with $X$ in $J$.

Theorem 3.3 ([17, 19]). The Abel-Jacobi image $\nu^{*}$ equals the harmonic volume $\nu_{X}=2 I_{X}$.

This theorem and Lemma 3.2 give us 
Proposition 3.4. If there exists an $\omega \in \wedge^{3} H^{1} \cap\left(\wedge^{3} H^{1,0}+\wedge^{3} H^{0,1}\right)$ such that $\nu_{X}(\omega)$ is nonzero modulo $\mathbb{Z}$, then $W_{1}-W_{1}^{-} \notin \mathrm{CH}_{1}(J)_{\text {alg }}$, i.e., $W_{1}-W_{1}^{-}$is algebraically nontrivial in $J$.

We remark that $F^{3} H^{3}(J ; \mathbb{C})=\wedge^{3} H^{1,0}$. Similarly, we obtain a sufficient condition that $W_{k}-W_{k}^{-}$is algebraically nontrivial in $J$. See Faucette [9] and Otsubo [26].

\section{Pointed Torelli Theorem}

We explain the relationship between the pointed harmonic volume and the congruence group of an extension of mixed Hodge structures. The classical Torelli theorem states that a compact Riemann surface $X$ is determined by its Jacobian variety $J(X)$ regarded as a complex torus. We introduce an extension of this theorem for pointed compact Riemann surfaces. In order to state the theorem, we need to define a mixed Hodge structure on $\mathbb{Z}$-module of finite rank using Chen's iterated integrals. The pointed harmonic volume plays an important role for the proof of the above theorem. It determines the moduli space of pointed compact Riemann surfaces. In the latter half of this section, we give the higher Abel-Jacobi image $\Phi_{k}\left(W_{k}(x)-W_{k}^{ \pm}(y)\right)$. This suggests an algorithm in proving the nontriviality condition for the higher Ceresa cycles.

A mixed Hodge structure on a $\mathbb{Z}$-module $H_{\mathbb{Z}}$ of finite rank consists of two filtrations, an increasing weight filtration $W_{p} H_{\mathbb{Q}}$ and a decreasing Hodge filtration $F^{p} H_{\mathbb{C}}$ which induces a $\mathbb{Q}$-Hodge structure of weight- $k$ on each graded piece

$$
\mathrm{Gr}_{k}^{W} H_{\mathbb{Q}}=W_{k} H_{\mathbb{Q}} / W_{k-1} H_{\mathbb{Q}} .
$$

Here $H_{\mathbb{Q}}$ denotes $H_{\mathbb{Z}} \otimes_{\mathbb{Z}} \mathbb{Q}$. For mixed Hodge structures $A$ and $B$, let $\operatorname{Ext}(A, B)$ denote the group of congruence classes of extensions of mixed Hodge structures, i.e., exact sequences

$$
0 \longrightarrow B \stackrel{\beta}{\longrightarrow} H \stackrel{\alpha}{\longrightarrow} A \longrightarrow 0
$$

of mixed Hodge structures with the natural equivalence relation and Baer sum. If $v-w=-1$ for the weight $v$ and $w$ of Hodge structures $A$ and $B$ respectively, then we have the isomorphism

$$
\tau: \operatorname{Ext}(A, B) \cong J \operatorname{Hom}(A, B) .
$$

Let $E$ denote a congruence class of an extension of $A$ by $B$. Choose a retraction $r_{\mathbb{Z}}: H_{\mathbb{Z}} \rightarrow B_{\mathbb{Z}}$, i.e., $r_{\mathbb{Z}} \circ \beta=\operatorname{id}_{B_{\mathbb{Z}}}$ defined over $\mathbb{Z}$ and a section $s_{F}: A \rightarrow H$, i.e., $\alpha \circ s_{F}=\operatorname{id}_{A}$ which preserves the Hodge filtration. Then $\tau(E) \in J \operatorname{Hom}(A, B)$ is represented by $r_{\mathbb{C}} \circ s_{F} \in \operatorname{Hom}(A, B)_{\mathbb{C}}$. See Carlson [2] for the general case. 
We concentrate on the compact Riemann surface $X$. Using the augmentation ideal $J_{x}$ of $\mathbb{Z} \pi_{1}(X, x)$, we obtain the short exact sequence

$$
0 \longrightarrow J_{x}^{2} / J_{x}^{3} \longrightarrow J_{x} / J_{x}^{3} \longrightarrow J_{x} / J_{x}^{2} \longrightarrow 0
$$

The natural map

$$
\mathbb{Z} \pi_{1}(X, x) \ni \gamma \mapsto \gamma-1 \in J_{x}
$$

induces an isomorphism $H_{1} \cong J_{x} / J_{x}^{2}$ for any point $x \in X$. For the additive group $M, M^{*}$ denotes $\operatorname{Hom}_{\mathbb{Z}}(M, \mathbb{Z})$. It is known that $\left(J_{x}^{2} / J_{x}^{3}\right)^{*}$ is isomorphic to $K \subset H^{1} \otimes H^{1}$ which is the kernel of $():, H^{1} \otimes H^{1} \rightarrow \mathbb{Z}$ induced by the intersection pairing. We obtain the dual short exact sequence

$$
0 \longrightarrow H^{1} \stackrel{\alpha}{\longrightarrow}\left(J_{x} / J_{x}^{3}\right)^{*} \stackrel{\beta}{\longrightarrow} K \longrightarrow 0
$$

From Corollary 2.9, $\left(J_{x} / J_{x}^{3}\right)^{*}$ is regarded as a sublattice of $H^{0}\left(\bar{B}_{2}(X), x\right)$. In this situation, the image $\alpha(\omega)$ is a linear functional $\int \omega$, where $\omega \in H^{1}$ is a real harmonic 1-form with integral periods. We give the homomorphism $\beta$ as follows. Let $\varphi \in\left(J_{x} / J_{x}^{3}\right)^{*}$ be an element. Take a homotopy functional $\bar{\varphi}$ on $\mathbb{Z} \pi_{1}(X, x)$ as in (2.2). Here $a_{i}, b_{i} \in H^{1}$. We set $\beta(\varphi)=\sum a_{i} \otimes b_{i} \in K$.

In the general case, Hain [13] defined a mixed Hodge structure on the truncation of the fundamental group ring of complex manifolds. We explain the mixed Hodge structure on $\left(J_{x} / J_{x}^{3}\right)^{*}$ as a sublattice of $H^{0}\left(\bar{B}_{2}(X), x\right)$. For $i=0,1,2$, the weight filtration $W_{i}$ is obtained by $H^{0}\left(\bar{B}_{i}(X), x\right)$. Here $W_{i}$ is defined over $\mathbb{Q}$. The Hodge filtration $F^{p}$ is induced by $n$ holomorphic 1 -forms on $X$ with $n \geq p$ in the linear functional of $H^{0}\left(\bar{B}_{2}(X), x\right)$ defined over $\mathbb{C}$. See Pulte [28] for details. Using Poincaré duality, Pulte [28, Lemma 3.7] obtained the isomorphism of Hodge structures

$$
\operatorname{Hom}\left(K, H^{1}\right) \rightarrow \operatorname{Hom}\left(K \otimes H^{1}, \mathbb{C}\right)
$$

By combining (4.1) and this isomorphism, Lemma 3.1 gives us

Theorem 4.1 ([28, Theorem 3.9]). We have an isomorphism

$$
\operatorname{Ext}\left(K, H^{1}\right) \ni m_{x} \mapsto I_{(X, x)} \in \operatorname{Hom}_{\mathbb{Z}}\left(K \otimes H^{1}, \mathbb{R} / \mathbb{Z}\right) .
$$

The Abel-Jacobi image $\nu^{*}=\Phi_{1}\left(W_{1}(x)-W_{1}^{ \pm}(y)\right)$ is an element of the intermediate Jacobian $J_{1}(J)=\operatorname{Hom}_{\mathbb{Z}}\left(\wedge^{3} H^{1}, \mathbb{R} / \mathbb{Z}\right)$. A natural homomorphism $K \otimes H^{1} \rightarrow \wedge^{3} H^{1}$ induces an injective homomorphism

$$
\operatorname{Hom}_{\mathbb{Z}}\left(\wedge^{3} H^{1}, \mathbb{R} / \mathbb{Z}\right) \rightarrow \operatorname{Hom}_{\mathbb{Z}}\left(K \otimes H^{1}, \mathbb{R} / \mathbb{Z}\right) .
$$

Pulte extended Theorem 3.3 . 
Theorem 4.2 ([28, Theorem 4.9]). The above injection is given by

$$
\Phi_{1}\left(W_{1}(x)-W_{1}^{ \pm}(y)\right) \mapsto I_{(X, x)} \mp I_{(X, y)} .
$$

As an application of the pointed harmonic volume for $(X, x)$, Pulte proved the following pointed Torelli theorem.

Theorem 4.3 ([28, Theorem 5.5]). Suppose that $X$ and $Y$ are compact Riemann surfaces and that $x \in X$ and $y \in Y$. With the possible exception of two points $x$ in $X$, if there is a ring isomorphism

$$
\mathbb{Z} \pi_{1}(X, x) / J_{x}^{3} \rightarrow \mathbb{Z} \pi_{1}(Y, y) / J_{y}^{3}
$$

which preserves the mixed Hodge structure, then there is a biholomorphism $\varphi: X \rightarrow Y$ such that $\varphi(x)=y$. If $X$ is hyperelliptic or if the harmonic volume is nonzero, then there are no exceptional points.

Pointed harmonic volumes determine the structure of pointed compact Riemann surfaces. In the proof of this theorem, the classical Torelli theorem follows from the equivalence of the exact sequence (4.2), which preserves the mixed Hodge structure, and therefore we obtain the biholomorphism $X \cong Y$. Pulte noted that the pointed Torelli theorem can fail for very special curves where the harmonic volume $\nu_{X}$ is zero but the curve is nonhyperelliptic. The zero locus of the harmonic volume for nonhyperelliptic curves is unknown.

Let $k$ be an integer such that $1 \leq k \leq g-2$. Otsubo [26. Proposition 3.7] obtained a reduction of the higher Abel-Jacobi image $\Phi_{k}\left(W_{k}(x)-W_{k}^{ \pm}(y)\right) \in$ $J_{k}(J)$ to the case $k=1$, using the commutative diagram (2.4). We recall $J_{k}(J) \cong \operatorname{Hom}\left(\wedge^{2 k+1} H^{1}, \mathbb{R} / \mathbb{Z}\right)$.

Proposition 4.4. Suppose that $\omega_{i} \in H^{1}$ for $1 \leq i \leq 2 k+1$. We have

$$
\begin{aligned}
& k ! \Phi_{k}\left(W_{k}(x)-W_{k}^{ \pm}(y)\right)\left(\omega_{1} \wedge \omega_{2} \wedge \cdots \wedge \omega_{2 k+1}\right) \\
& \quad=k ! \sum_{\sigma} \Phi_{1}\left(W_{1}(x)-W_{1}^{ \pm}(y)\right)\left(\omega_{\sigma(1)} \wedge \omega_{\sigma(2)} \wedge \omega_{\sigma(3)}\right) \prod_{i=1}^{k-1}\left(\omega_{\sigma(2 i+2)}, \omega_{\sigma(2 i+3)}\right),
\end{aligned}
$$

where $\sigma$ runs through the elements of the (2k+1)-th symmetric group $S_{2 k+1}$ such that

$$
\sigma(1)<\sigma(2)<\sigma(3), \sigma(2 i+2)<\sigma(2 i+3) \text { for } 1 \leq i \leq k-1,
$$

and

$$
\sigma(2 i+2)<\sigma(2 i+4) \text { for } 1 \leq i \leq k-2 .
$$

Faucette [8] defined the higher-dimensional harmonic volume as follows. Suppose $g \geq 2 k+1$ for a fixed natural number $k$. Let $\omega_{1}, \omega_{2}, \ldots, \omega_{2 k+1} \in H^{1}$ 
be real harmonic 1 -forms on $X$ with $\mathbb{Z}$-periods. A homomorphism $A_{k}: X_{k} \rightarrow$ $\mathbb{R}^{2 k+1} / \mathbb{Z}^{2 k+1}$ is defined by

$$
A_{k}\left(x_{1}, x_{2}, \ldots, x_{k}\right)=\left(\sum_{i=1}^{k} \int_{x}^{x_{i}} \omega_{1}, \ldots, \sum_{i=1}^{k} \int_{x}^{x_{i}} \omega_{2 k+1}\right) .
$$

We define $\omega=\omega_{1} \wedge \omega_{2} \wedge \ldots \wedge \omega_{2 k+1} \in \wedge^{2 k+1} H^{1}$ to be a good form on $J$ if there exists a topological $(2 k+1)$-chain $C_{2 k+1}$ in $\mathbb{R}^{2 k+1} / \mathbb{Z}^{2 k+1}$ whose boundary is the image $A_{k}\left(X_{k}\right)$. The higher-dimensional harmonic volume $I_{X}^{k}$ is defined to be the linear functional on the good forms with values in $\mathbb{R} / \mathbb{Z}$

$$
I_{X}^{k}(\omega):=\int_{C_{2 k+1}} d x_{1} \wedge d x_{2} \wedge \cdots \wedge d x_{2 k+1} \quad \bmod \mathbb{Z},
$$

where the $\omega_{i}$ is the pullback of $d x_{i}$ by $A_{k}$. The definition is independent of the choice of base point $x$. Faucette proved that $\Phi_{k}\left(W_{k}-W_{k}^{-}\right)$equals $2 I_{X}^{k}$ as a linear functional on good forms. In 8 , he evaluated $I_{X}^{2}$ for an unramified double covering of the Fermat quartic $F_{4}$, and proved that the second Ceresa cycle $W_{2}-W_{2}^{-}$for the curve is algebraically nontrivial.

\section{Nontrivial algebraic cycles in the Jacobian varieties}

In Section 3, we give a sufficient condition for the $k$-th Ceresa cycle $W_{k}-W_{k}^{-} \in$ $\mathrm{CH}_{k}(J)_{\text {hom }}$ to be algebraically nontrivial. Harris [18] proved the nontriviality for the Fermat quartic $F_{4}$. Bloch [1] further studied the Fermat quartic $F_{4}$ in the context of number theory. Using methods similar to Harris, the author [33, 34] proved the nontriviality for the Klein quartic $K_{4}$ and Fermat sextic $F_{6}$. Otsubo [26] extended these results using number theory and obtained an algorithm in proving the nontriviality for the $N$-th Fermat curve $F_{N}$ for $N \geq$ 4. Using his method, we recently obtained the nontriviality for some cyclic quotients of $F_{N}$ 35. We remark that all the above curves have a representation as a branched cyclic covering of $\mathbb{C} P^{1}$ and certain values of the harmonic volume for this kind of curve are known to be computable. Nevertheless, all the values of the harmonic volume for a specific curve are unknown.

\subsection{Fermat curves}

We give some value of the higher Abel-Jacobi image $\Phi_{k}\left(W_{k}-W_{k}^{-}\right)$for the Fermat curve as an iterated integral form. In the former half of this subsection, we have compiled some basic facts on the Fermat curve.

For $N \in \mathbb{Z}_{\geq 4}$, let $F_{N}=\left\{(X: Y: Z) \in \mathbb{C} P^{2} ; X^{N}+Y^{N}=Z^{N}\right\}$ denote the Fermat curve of degree $N$, a compact Riemann surface of genus $(N-1)(N-$ 
2)/2. Let $x$ and $y$ denote $X / Z$ and $Y / Z$ respectively. The equation $X^{N}+$ $Y^{N}=Z^{N}$ induces $x^{N}+y^{N}=1$. Let $\zeta$ denote $\exp (2 \pi \sqrt{-1} / N)$. Holomorphic automorphisms $\alpha$ and $\beta$ of $F_{N}$ are defined as $\alpha(X: Y: Z)=(\zeta X: Y: Z)$ and $\beta(X: Y: Z)=(X: \zeta Y: Z)$ respectively. Let $\mu_{N}$ be the group of $N$-th roots of unity in $\mathbb{C}$. We have $\alpha \beta=\beta \alpha$ and the subgroup, denoted by $G$, of holomorphic automorphisms of $F_{N}$ generated by $\alpha$ and $\beta$ is isomorphic to $\mu_{N} \times \mu_{N}$. Let $\gamma_{0}$ be a path $[0,1] \ni t \mapsto\left(t, \sqrt[N]{1-t^{N}}\right) \in F(N)$, where $\sqrt[N]{1-t^{N}}$ is a real nonnegative analytic function on $[0,1]$. A loop in $F_{N}$ is defined by

$$
\kappa_{0}=\gamma_{0} \cdot\left(\beta \gamma_{0}\right)^{-1} \cdot\left(\alpha \beta \gamma_{0}\right) \cdot\left(\alpha \gamma_{0}\right)^{-1},
$$

where the product $\ell_{1} \cdot \ell_{2}$ indicates that we traverse $\ell_{1}$ first, then $\ell_{2}$. We consider a loop $\alpha^{i} \beta^{j} \kappa_{0}$ as an element of the first homology group $H_{1}\left(F_{N} ; \mathbb{Z}\right)$ of $F_{N}$, which is well-known to be a cyclic $G$-module [12, Appendix].

Let $\mathbf{I}$ be an index set $\left\{(a, b) \in(\mathbb{Z} / N \mathbb{Z})^{\oplus 2} ; a, b, a+b \neq 0\right\}$. For $a \in \mathbb{Z} / N \mathbb{Z} \backslash$ $\{0\}$, we denote its representative by $\langle a\rangle \in\{1,2, \ldots, N-1\}$. A differential 1 -form on $F_{N}$ is defined by

$$
\omega_{0}^{a, b}=x^{\langle a\rangle-1} y^{\langle b\rangle-1} d x / y^{N-1} .
$$

Set $\mathbf{I}_{\text {holo }}=\{(a, b) \in \mathbf{I} ;\langle a\rangle+\langle b\rangle<N\}$. It is well-known that

$$
\left.\left\{\omega_{0}^{a, b} ;(a, b) \in \mathbf{I}\right\}\right\},\left\{\omega_{0}^{a, b} ;(a, b) \in \mathbf{I}_{\text {holo }}\right\} \text {, and }\left\{\omega_{0}^{-a,-b} ;(a, b) \in \mathbf{I}_{\text {holo }}\right\},
$$

are bases of $H^{1}(F(N) ; \mathbb{C}), H^{1,0}(F(N))$, and $H^{0,1}(F(N))$, respectively. See Lang [21] for example. Clearly,

$$
\int_{\alpha^{i} \beta^{j} \gamma_{0}} \omega_{0}^{a, b}=\zeta^{a i+b j} \int_{\gamma_{0}} \omega_{0}^{a, b}=\zeta^{a i+b j} \frac{B(\langle a\rangle / N,\langle b\rangle / N)}{N} .
$$

The beta function $B(u, v)$ is defined by $\int_{0}^{1} t^{u-1}(1-t)^{v-1} d t$ for $u, v>0$. We set $B_{a, b}^{N}=B(\langle a\rangle / N,\langle b\rangle / N)$. The integral of $\omega_{0}^{a, b}$ along $\alpha^{i} \beta^{j} \kappa_{0}$ is obtained as follows,

Proposition 5.1 ([12, Appendix]). We have

$$
\int_{\alpha^{i} \beta^{j} \kappa_{0}} \omega_{0}^{a, b}=B_{a, b}^{N}\left(1-\zeta^{a}\right)\left(1-\zeta^{b}\right) \zeta^{a i+b j} / N
$$

We denote the 1-form $N \omega_{0}^{a, b} / B_{a, b}^{N}$ by $\omega^{a, b}$. This implies $\int_{\alpha^{i} \beta^{j} \kappa_{0}} \omega^{a, b} \in \mathbb{Z}[\zeta]$.

Let $\mathbb{Q}\left(\mu_{N}\right)$ be the $N$-th cyclotomic field, and $\mathcal{O}$ its integer ring. For a $\mathbb{Z}$-module $M$, we denote the $\mathcal{O}$-module $M_{\mathcal{O}}$ by $M \otimes_{\mathbb{Z}} \mathcal{O}$. For each embedding $\sigma: \mathbb{Q}\left(\mu_{N}\right) \hookrightarrow \mathbb{C}$, let $h \in(\mathbb{Z} / N \mathbb{Z})^{*}$ be the element such that $\sigma(\xi)=\zeta^{h}$, where $\xi$ is a primitive $N$-th root of unity. Proposition 5.1 gives us the element 
$\varphi^{a, b} \in H_{\mathcal{O}}$ whose image by $H_{\mathcal{O}} \subset H_{\mathbb{Q}\left(\mu_{N}\right)} \hookrightarrow H_{\mathbb{C}}$ is $\omega^{h a, h b}$. In a straightforward manner, we obtain the intersection pairing.

Proposition 5.2 ([26, Proposition 4.2]).

$$
\left(\varphi^{a, b}, \varphi^{c, d}\right)= \begin{cases}N^{2} \frac{\left(1-\xi^{a}\right)\left(1-\xi^{b}\right)}{1-\xi^{a+b}} & \text { if }(a, b)=(-c,-d), \\ 0 & \text { otherwise. }\end{cases}
$$

It immediately follows that $\varphi^{a, b} \otimes \varphi^{c, d} \in K_{\mathcal{O}} \subset H_{\mathcal{O}}^{\otimes 2}$ if only and if $(a, b) \neq$ $(-c,-d)$.

The harmonic volume naturally extends to

$$
\nu_{\mathcal{O}}=2 I_{\mathcal{O}}:\left(\wedge^{3} H\right)_{\mathcal{O}}^{\prime} \rightarrow(\mathcal{O} \otimes \mathbb{R}) / \mathcal{O}
$$

We have a natural isomorphism

$$
\mathcal{O} \otimes \mathbb{R} \cong\left[\prod_{\sigma: \mathbb{Q}\left(\mu_{N}\right) \hookrightarrow \mathbb{C}} \mathbb{C}\right]^{+}
$$

where $\sigma$ runs through the embedding of $K$ into $\mathbb{C}$ and + denotes the fixed part under complex conjugation acting on both $\{\sigma\}$ and $\mathbb{C}$ at the same time. Let $\nu_{\sigma}$ denote the $\sigma$-component of the harmonic volume $\nu_{\mathcal{O}}$. Let $\operatorname{Tr}:(\mathcal{O} \otimes \mathbb{R}) / \mathcal{O} \rightarrow$ $\mathbb{R} / \mathbb{Z}$ be the trace map. We obtain $\operatorname{Tr} \circ \nu_{\mathcal{O}}=\sum_{\sigma: \mathbb{Q}\left(\mu_{N}\right) \hookrightarrow \mathbb{C}} \nu_{\sigma}$. To prove the nonvanishing of $\nu_{\mathcal{O}}$, it is sufficient to prove that of $\operatorname{Tr} \circ \nu_{\mathcal{O}}$. We restrict ourselves to the following situation.

Assumption 5.3. The elements $\left(a_{i}, b_{i}\right) \in \mathbf{I}(i=1,2,3)$ satisfy the following conditions:

(1) $\sum_{i=1}^{3}\left(a_{i}, b_{i}\right)=(0,0)$.

(2) For any $h \in(\mathbb{Z} / N \mathbb{Z})^{*}$, we have either $\left(h a_{i}, h b_{i}\right) \in \mathbf{I}_{\text {holo }}$ for $i=1,2$, or $\left(h a_{i}, h b_{i}\right) \notin \mathbf{I}_{\text {holo }}$ for $i=1,2$.

From the second assumption, the correction term $\eta$ in the harmonic volume becomes zero. Then we have only to compute the iterated integral part. Using the first assumption and [34, Theorem 3.6], Otsubo [26] proved

Theorem 5.4. Under Assumption 5.3, we obtain

$$
\operatorname{Tr} \circ \nu_{\mathcal{O}}\left(\frac{\varphi^{a_{1}, b_{1}} \otimes \varphi^{a_{2}, b_{2}} \otimes \varphi^{a_{3}, b_{3}}}{\left(1-\xi^{-a_{3}}\right)\left(1-\xi^{-b_{3}}\right)}\right)=2 N^{2} \sum \int_{\gamma_{0}} \omega^{h a_{1}, h b_{1}} \omega^{h a_{2}, h b_{2}},
$$

where the sum is taken over $h \in(\mathbb{Z} / N \mathbb{Z})^{*}$ such that $\left(h a_{i}, h b_{i}\right) \in \mathbf{I}_{\text {holo for }}$ $i=1,2$. 
We take an element $\varphi=\varphi_{1} \wedge \varphi_{2} \wedge \cdots \wedge \varphi_{2 k+1} \in \wedge^{2 k+1} H_{\mathcal{O}}$ for $1 \leq k \leq g-2$ in the following way. Let $\left(a_{i}, b_{i}\right) \in \mathbf{I}(1 \leq i \leq 2 k+1)$ be distinct elements such that $\left(a_{i}, b_{i}\right)(i=1,2,3)$ satisfy Assumption 5.3 and $\left(a_{2 i+2}, b_{2 i+2}\right)=$ $\left(-a_{2 i+3},-b_{2 i+3}\right)$ for $1 \leq i \leq k-1$. We let

$$
\varphi_{1}=\varphi^{a_{1}, b_{1}}, \varphi_{2}=\varphi^{a_{2}, b_{2}}, \varphi_{3}=\frac{1}{\left(1-\xi^{-a_{3}}\right)\left(1-\xi^{-b_{3}}\right)} \varphi^{a_{3}, b_{3}},
$$

and

$$
\varphi_{2 i+2}=\frac{1-\xi^{a_{2 i+2}+b_{2 i+2}}}{\left(1-\xi^{-a_{2 i+2}}\right)\left(1-\xi^{-b_{2 i+2}}\right)} \varphi^{a_{2 i+2}, b_{2 i+2}}, \varphi_{2 i+3}=\varphi^{a_{2 i+3}, b_{2 i+3}}
$$

for $1 \leq i \leq k-1$. Set $\varphi=\varphi_{1} \wedge \varphi_{2} \wedge \cdots \wedge \varphi_{2 k+1}$. Proposition 5.2 induces $\left(\varphi_{2 i+2}, \varphi_{2 i+3}\right)=N^{2}$. Theorem 5.4 and Proposition 4.4 give us

Corollary 5.5. For $1 \leq k \leq g-2$, we have

$$
k ! \operatorname{Tr} \circ \Phi_{k}\left(W_{k}-W_{k}^{-}\right)(\varphi)=k ! 2 N^{2 k} \sum \int_{\gamma_{0}} \omega^{h a_{1}, h b_{1}} \omega^{h a_{2}, h b_{2}},
$$

where the sum is as in Theorem 5.4.

\subsection{A generalized hypergeometric function}

For the numerical calculation, we recall the generalized hypergeometric function ${ }_{3} F_{2}$. The higher Abel-Jacobi image in the previous subsection is presented by the special values of ${ }_{3} F_{2}$. We introduce the condition to prove nontriviality for the Ceresa cycle $W_{k}-W_{k}^{-}$for $F_{N}$.

The gamma function is defined as $\Gamma(\tau)=\int_{0}^{\infty} e^{-t} t^{\tau-1} d t$ for $\tau>0$ and the Pochhammer symbol as $(\alpha, n)=\Gamma(\alpha+n) / \Gamma(\alpha)$ for any nonnegative integer $n$. For $x \in\{z \in \mathbb{C} ;|z|<1\}$ and $\beta_{1}, \beta_{2} \notin\{0,-1,-2, \ldots\}$, the generalized hypergeometric function ${ }_{3} F_{2}$ is defined by

$$
{ }_{3} F_{2}\left(\begin{array}{c}
\alpha_{1}, \alpha_{2}, \alpha_{3} \\
\beta_{1}, \beta_{2}
\end{array} ; x\right)=\sum_{n=0}^{\infty} \frac{\left(\alpha_{1}, n\right)\left(\alpha_{2}, n\right)\left(\alpha_{3}, n\right)}{\left(\beta_{1}, n\right)\left(\beta_{2}, n\right)(1, n)} x^{n} .
$$

Its radius of convergence is 1 . However, if $\operatorname{Re}\left(\beta_{1}+\beta_{2}-\alpha_{1}-\alpha_{2}-\alpha_{3}\right)>0$, then ${ }_{3} F_{2}$ converges when $|x|=1$.

Proposition 5.6. For $i=1,2$, we put the 1 -forms $\omega_{i}=t^{\alpha_{i}-1}(1-t)^{\beta_{i}-1} d t$ on the unit interval $[0,1]$. If $0<\alpha_{i}, \beta_{i}<1$ for each $i$, then we have the iterated integral

$$
\int_{[0,1]} \omega_{1} \omega_{2}=\frac{B\left(\alpha_{1}+\alpha_{2}, \beta_{2}\right)}{\alpha_{1}}{ }_{3} F_{2}\left(\begin{array}{c}
\alpha_{1}, 1-\beta_{1}, \alpha_{1}+\alpha_{2} \\
1+\alpha_{1}, \alpha_{1}+\alpha_{2}+\beta_{2}
\end{array} ; 1\right) .
$$


The proof of this computation is straightforward. See [34, Proposition 3.7] for example.

For simplicity, we introduce

$$
\Gamma\left(\begin{array}{c}
a_{1}, a_{2}, \ldots, a_{n} \\
b_{1}, b_{2}, \ldots, b_{m}
\end{array}\right)=\frac{\Gamma\left(a_{1}\right) \Gamma\left(a_{2}\right) \cdots \Gamma\left(a_{n}\right)}{\Gamma\left(b_{1}\right) \Gamma\left(b_{2}\right) \cdots \Gamma\left(b_{m}\right)} .
$$

Using Dixon's formula [30] and Proposition 5.6. Otsubo obtained another representation of the above iterated integral $\int_{[0,1]} \omega_{1} \omega_{2}$ given as

$$
\Gamma\left(\begin{array}{c}
\alpha_{1}, \beta_{2}, \alpha_{1}+\alpha_{2}, \beta_{1}+\beta_{2} \\
\alpha_{1}+\alpha_{2}+\beta_{2}, \alpha_{1}+\beta_{1}+\beta_{2}
\end{array}\right){ }_{3} F_{2}\left(\begin{array}{cc}
\alpha_{1}, \beta_{2}, \alpha_{1}+\alpha_{2}+\beta_{1}+\beta_{2} \\
\alpha_{1}+\alpha_{2}+\beta_{2}, \alpha_{1}+\beta_{1}+\beta_{2}
\end{array} ; 1\right) .
$$

This induces the computation of iterated integrals of 1 -forms on $F_{N}$.

Proposition 5.7. Suppose that $\left(a_{i}, b_{i}\right) \in \mathbf{I}$. Let $\alpha_{i}$ and $\beta_{i}$ denote $\left\langle a_{i}\right\rangle / N$ and $\left\langle b_{i}\right\rangle / N$, respectively. Then the iterated integral $\int_{\gamma_{0}} \omega^{a_{1}, b_{1}} \omega^{a_{2}, b_{2}}$ obtains in the form

$\Gamma\left(\begin{array}{c}\alpha_{1}+\alpha_{2}, \beta_{1}+\beta_{2}, \alpha_{1}+\beta_{1}, \alpha_{2}+\beta_{2} \\ \alpha_{2}, \beta_{1}, \alpha_{1}+\alpha_{2}+\beta_{2}, \alpha_{1}+\beta_{1}+\beta_{2}\end{array}\right){ }_{3} F_{2}\left(\begin{array}{cc}\alpha_{1}, \beta_{2}, \alpha_{1}+\alpha_{2}+\beta_{1}+\beta_{2} \\ \alpha_{1}+\alpha_{2}+\beta_{2}, \alpha_{1}+\beta_{1}+\beta_{2}\end{array} ; 1\right)$.

Put

$$
\left(a_{1}, b_{1}\right)=(1,-2),\left(a_{2}, b_{2}\right)=(-2,1), \text { and }\left(a_{3}, b_{3}\right)=(1,1) .
$$

These $\left(a_{i}, b_{i}\right)$ 's satisfy Assumption 5.3. Moreover, we have either $\left(h a_{i}, h b_{i}\right) \in$ $\mathbf{I}_{\text {holo }}$ for $i=1,2,3$, or $\left(h a_{i}, h b_{i}\right) \notin \mathbf{I}_{\text {holo }}$ for $i=1,2,3$. It immediately follows that

$$
\varphi \in\left(\wedge^{2 k+1} H \cap\left(F^{k+2}+\overline{F^{k+2}}\right)\right) \otimes_{\mathbb{Z}} \mathcal{O}
$$

for any $h \in(\mathbb{Z} / N \mathbb{Z})^{*}$. From the above proposition, we have

$$
\int_{\gamma_{0}} \omega^{h,-2 h} \omega^{-2 h, h}=\frac{\Gamma\left(1-\frac{h}{N}\right)^{4}}{\Gamma\left(1-\frac{2 h}{N}\right)^{4}}{ }_{3} F_{2}\left(\begin{array}{c}
\frac{h}{N}, \frac{h}{N}, 1-\frac{2 h}{N} \\
1,1
\end{array} ; 1\right)
$$

for $h \in(\mathbb{Z} / N \mathbb{Z})^{*}$. Using this and Corollary 5.5, we have

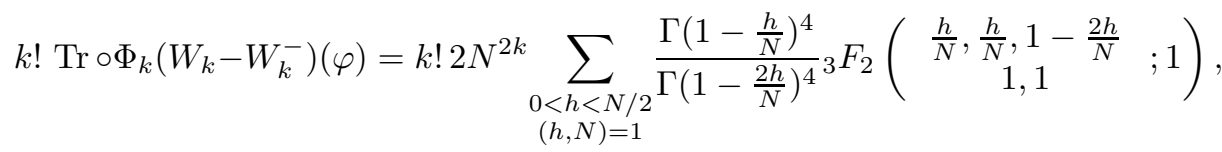

for $1 \leq k \leq N-2$. This value is denoted by $f(N, k)$. Lemma 3.2 and Condition (5.1) induce an algebraically nontrivial condition on the $k$-th Ceresa cycle $k !\left(W_{k}-W_{k}^{-}\right) \in \mathrm{CH}_{k}(J)_{\text {hom }}$.

Theorem 5.8. For the $N$-th Fermat curve $F_{N}$ and integer $k$ such that $1 \leq$ $k \leq(N-3) / 2$, if the value $f(N, k)$ is noninteger, then $k !\left(W_{k}-W_{k}^{-}\right)$is not algebraically equivalent to zero in $J\left(F_{N}\right)$. 
This indeed holds for $N \leqq 1000, k=1$, or $N \leqq 8$ and any $k$, following numerical calculations using Mathematica. Otsubo pointed out a sufficient condition for nontorsion. If $\int_{\gamma_{0}} \omega^{h,-2 h} \omega^{-2 h, h}$ is not an element of $\mathbb{Q}\left(\mu_{N}\right)$ for some integer $h$ with $0<h<N / 2$ and $(h, N)=1$, then $W_{k}-W_{k}^{-} \in \mathrm{CH}_{k}(J)_{\text {alg }}$ is nontorsion.

\subsection{Cyclic quotients of Fermat curves}

As an extension of Otsubo's result, we give some value of the higher AbelJacobi image $\Phi_{k}\left(W_{k}-W_{k}\right)$ for a cyclic quotient of a Fermat curve.

For any prime number $N$ such that $N \geq 5$, we define a cyclic quotient of a Fermat curve $C_{N}^{a, b}$ as the projective curve whose affine equation is

$$
C_{N}^{a, b}:=\left\{(u, v) \in \mathbb{C}^{2} ; v^{N}=u^{a}(1-u)^{b}\right\},
$$

where integers $a$ and $b$ are coprime and satisfy $0<a, b<N$. The curve $C_{N}^{a, b}$ is a compact Riemann surface of genus $(N-1) / 2$. We denote by $\pi: F_{N} \rightarrow C_{N}^{a, b}$ the $N$-fold unramified covering $\pi(x, y)=(u, v)=\left(x^{N}, x^{a} y^{b}\right)$. The curve $C_{7}^{1,2}$ is called the Klein quartic $K_{4}$. There is a unique 1 -form $\tau^{\alpha, \beta}$ on $C_{N}^{a, b}$ such that $\pi^{*} \tau^{\alpha, \beta}=\omega^{\alpha, \beta}$. Then $\left\{\tau^{\lambda a, \lambda b}\right\}_{\langle\lambda a\rangle+\langle\lambda a\rangle<N}$ is a basis of $H^{1,0}$ of $C_{N}^{a, b}$ for an integer $\lambda$ and the above fixed $a$ and $b$. See Lang [21] for example. For an embedding $\sigma: K \ni \xi \mapsto \zeta^{h} \in \mathbb{C}$, we can choose the unique 1-form $\eta^{\alpha, \beta}$ on $C_{N}^{a, b}$ so that $\sigma\left(\eta^{\alpha, \beta}\right)=\tau^{h \alpha, h \beta}$.

For the rest of this paper, we assume that $N$ is prime with $N=1$ modulo 3. There exists an integer $1<m<N-1$ such that $m^{2}+m+1=0$ modulo $N$. Set

$$
\left(a_{1}, b_{1}\right)=(1, m),\left(a_{2}, b_{2}\right)=\left(m, m^{2}\right), \text { and }\left(a_{3}, b_{3}\right)=\left(m^{2}, 1\right) .
$$

Lemma 5.9. These $\left(a_{i}, b_{i}\right)$ 's satisfy Assumption 5.3. Furthermore, the conditions $\left(h a_{i}, h b_{i}\right) \in \mathbf{I}_{\text {holo }}, i=1,2,3$ are equivalent.

Put

$$
\eta_{m}=\frac{\eta^{1, m} \wedge \eta^{m, m^{2}} \wedge \eta^{m^{2}, 1}}{\left(1-\xi^{-m^{2}}\right)\left(1-\xi^{-1}\right)}
$$

We have

$$
\eta_{m} \in\left(\wedge^{3} H \cap\left(F^{3}+\overline{F^{3}}\right)\right) \otimes_{\mathbb{Z}} \mathcal{O} .
$$

Theorem 5.4 gives us 
Proposition 5.10. The value of the harmonic volume for $C_{N}^{1, m}$ is

$$
\operatorname{Tr} \circ \nu_{\mathcal{O}}\left(\eta_{m}\right)=2 N^{3} \sum \int_{\gamma_{0}} \omega^{h, h m} \omega^{h m, h m^{2}},
$$

where the sum is taken over $h \in(\mathbb{Z} / N \mathbb{Z})^{*}$ such that $\left(h a_{i}, h b_{i}\right) \in \mathbf{I}_{\text {holo }}$ for $i=1,2,3$.

Proposition 5.11.

$$
\int_{\gamma_{0}} \omega^{h, h m} \omega^{h m, h m^{2}}=\Gamma\left(\begin{array}{c}
\frac{N-h}{N}, \frac{N-\left\langle h m^{2}\right\rangle}{N} \\
\frac{\langle h m\rangle}{N}
\end{array}\right)_{3}^{2} F_{2}\left(\begin{array}{c}
\frac{h}{N}, \frac{\langle h m\rangle}{N}, \frac{\left\langle h m^{2}\right\rangle}{N} \\
1,1
\end{array}\right)
$$

for an integer $h$ such that $0<h<N$ and $h+\langle h m\rangle+\left\langle h m^{2}\right\rangle=N$.

Similar to Theorem 5.8, we obtain the higher Abel-Jacobi image $\Phi_{k}\left(W_{k}-\right.$ $\left.W_{k}\right)(\varphi)$ and the sufficient condition that $W_{k}-W_{k}^{-}$is not algebraically equivalent to zero in $J\left(C_{N}^{1, m}\right)$. See 35 for details.

Acknowledgements. First of all, the author is grateful to Athanase Papadopoulos for valuable advice. He also thanks Subhojoy Gupta, Takuya Sakasai, and Kokoro Tanaka for reading the manuscript and giving useful comments. This work was partially supported by Fellowship for Research Abroad of Institute from the National Colleges of Technology and Grant-in-Aid for Young Scientists(B) (Grant No. 25800053). Part of the work was also done while the author stayed at the Danish National Research Foundation Centre of Excellence, QGM (Centre for Quantum Geometry of Moduli Spaces) in Aarhus University. He is very grateful for the warm hospitality of QGM. Finally he would like to thank the referee for valuable comments on the preliminary version of this chapter.

\section{References}

[1] S. Bloch: Algebraic cycles and values of L-functions. J. Reine Angew. Math., Vol. 350, pp. 94-108, 1984.

[2] J. A. Carlson: Extensions of mixed Hodge structures. Journees de geometrie algebrique, Angers/France 1979, pp. 107-127, 1980.

[3] G. Ceresa: C is not algebraically equivalent to $C^{-}$in its Jacobian. Ann. Math. (2), Vol. 117, pp. 285-291, 1983.

[4] K. T. Chen: Integration of paths. A faithful representation of paths by noncommutative formal power series. Trans. Am. Math. Soc., Vol. 89, pp. 395-407, 1959. 
[5] K. T. Chen: Iterated integrals, fundamental groups and covering spaces. Trans. Am. Math. Soc., Vol. 206, pp. 83-98, 1975.

[6] K. T. Chen: Iterated path integrals. Bull. Am. Math. Soc., Vol. 83, pp. 831-879, 1977.

[7] H. M. Farkas; I. Kra: Riemann surfaces. 2nd ed. Graduate Texts in Mathematics. 71. New York etc.: Springer-Verlag. xvi, 363 p. , 1992.

[8] W. M. Faucette: Higher dimensional harmonic volume can be computed as an iterated integral. Can. Math. Bull., Vol. 35, No. 3, pp. 328-340, 1992.

[9] W. M. Faucette: Harmonic volume, symmetric products, and the Abel-Jacobi map. Trans. Am. Math. Soc., Vol. 335, No. 1, pp. 303-327, 1993.

[10] W. Fulton: Intersection theory. 2nd ed. Ergebnisse der Mathematik und ihrer Grenzgebiete. 3. Folge. 2. Berlin: Springer. xiii, 470 p. , 1998.

[11] P. A. Griffiths: On the periods of certain rational integrals. I, II. Ann. of Math. (2) 90 (1969), 460-495; ibid. (2), Vol. 90, pp. 496-541, 1969.

[12] B. H. Gross: On the periods of Abelian integrals and a formula of Chowla and Selberg. With an appendix by David E. Rohrlich. Invent. Math., Vol. 45, pp. 193-211, 1978.

[13] R. M. Hain: The geometry of the mixed Hodge structure on the fundamental group. Algebraic geometry, Proc. Summer Res. Inst., Brunswick/Maine 1985, part 2, Proc. Symp. Pure Math. 46, pp. 247-282, 1987.

[14] R. M. Hain: Iterated integrals and algebraic cycles: examples and prospects. In Contemporary trends in algebraic geometry and algebraic topology. Proceedings of the Wei-Liang Chow and Kuo-Tsai Chen memorial conference on algebraic geometry and algebraic topology, Tianjin, China, October 9-13, 2000, pp. 55118. River Edge, NJ: World Scientific, 2002.

[15] R. M. Hain; D. Reed: On the Arakelov geometry of moduli spaces of curves. J. Differ. Geom., Vol. 67, No. 2, pp. 195-228, 2004.

[16] B. Harris: Triple products, modular forms, and harmonic volumes. Contemp. Math., Vol. 13, pp. 287-295, 1982.

[17] B. Harris: Harmonic volumes. Acta Math., Vol. 150, pp. 91-123, 1983.

[18] B. Harris: Homological versus algebraic equivalence in a Jacobian (algebraic cycle/ integral/ Fermat curve). Proc. Natl. Acad. Sci. USA, Vol. 80, pp. 11571158, 1983.

[19] B. Harris: Iterated integrals and cycles on algebraic manifolds. Nankai Tracts in Mathematics 7. River Edge, NJ: World Scientific. xii, 108 p. , 2004.

[20] N. Kawazumi: Canonical 2-forms on the moduli space of Riemann surfaces. Papadopoulos, A. (ed.), Handbook of Teichmüller theory. Volume II. Zürich: European Mathematical Society (EMS). IRMA Lectures in Mathematics and Theoretical Physics 13, pp. 217-237, 2009.

[21] S. Lang: Introduction to Algebraic and Abelian Functions. 2nd ed. Graduate Texts in Mathematics, 89. New York - Heidelberg - Berlin: Springer-Verlag. IX, 
169 p., 1982.

[22] J. W. Morgan: The algebraic topology of smooth algebraic varieties. Publ. Math., Inst. Hautes Étud. Sci., Vol. 48, pp. 137-204, 1978.

[23] S. Morita: Characteristic classes of surface bundles. Bull. Am. Math. Soc., New Ser., Vol. 11, pp. 386-388, 1984.

[24] S. Morita: The extension of Johnson's homomorphism from the Torelli group to the mapping class group. Invent. Math., Vol. 111, No. 1, pp. 197-224, 1993.

[25] D. Mumford: Towards an enumerative geometry of the moduli space of curves. Arithmetic and geometry, Pap. dedic. I. R. Shafarevich, Vol. II: Geometry, Prog. Math. 36, pp. 271-328, 1983.

[26] N. Otsubo: On the Abel-Jacobi maps of Fermat Jacobians. Math. Z., Vol. 270, No. 1-2, pp. 423-444, 2012.

[27] K. H. Paranjape; V. Srinivas: Algebraic cycles. Adhikari, S. D. (ed.), Current trends in mathematics and physics. A tribute to Harish-Chandra. New Delhi: Narosa Publishing House. pp. 71-86, 1995.

[28] M. J. Pulte: The fundamental group of a Riemann surface: Mixed Hodge structures and algebraic cycles. Duke Math. J., Vol. 57, No. 3, pp. 721-760, 1988.

[29] R. Ree: Lie elements and an algebra associated with shuffles. Ann. Math. (2), Vol. 68, pp. 210-220, 1958.

[30] L. J. Slater: Generalized hypergeometric functions. Cambridge: At the University Press. XIII, 273 p. , 1966.

[31] Y. Tadokoro: The harmonic volumes of hyperelliptic curves. Publ. Res. Inst. Math. Sci., Vol. 41, No. 3, pp. 799-820, 2005.

[32] Y. Tadokoro: The pointed harmonic volumes of hyperelliptic curves with Weierstrass base point. Kodai Math. J., Vol. 29, No. 3, pp. 370-382, 2006.

[33] Y. Tadokoro: A nontrivial algebraic cycle in the Jacobian variety of the Klein quartic. Math. Z., Vol. 260, No. 2, pp. 265-275, 2008.

[34] Y. Tadokoro: A nontrivial algebraic cycle in the Jacobian variety of the Fermat sextic. Tsukuba J. Math., Vol. 33, No. 1, pp. 29-38, 2009.

[35] Y. Tadokoro: Nontrivial algebraic cycles in the Jacobian varieties of some quotients of Fermat curves, Internat. J. Math, to appear.

[36] A. Weil: Foundations of algebraic geometry. American Mathematical Society Colloquium Publications. Vol. XXIX. Revised and enlarged edition. Providence, R.I.: American Mathematical Society (AMS). XX, 363 p. , 1962. 